Guram Bezhanishvili

NiCK BEZHANISHVILI

JULIA ILIN

\title{
Cofinal Stable Logics
}

\begin{abstract}
We generalize the $(\wedge, \vee)$-canonical formulas to $(\wedge, \vee)$-canonical rules, and prove that each intuitionistic multi-conclusion consequence relation is axiomatizable by $(\wedge, \vee)$-canonical rules. This yields a convenient characterization of stable superintuitionistic logics. The $(\wedge, \vee)$-canonical formulas are analogues of the $(\wedge, \rightarrow)$-canonical formulas, which are the algebraic counterpart of Zakharyaschev's canonical formulas for superintuitionistic logics (si-logics for short). Consequently, stable si-logics are analogues of subframe si-logics. We introduce cofinal stable intuitionistic multi-conclusion consequence relations and cofinal stable si-logics, thus answering the question of what the analogues of cofinal subframe logics should be. This is done by utilizing the $(\wedge, \vee, \neg)$-reduct of Heyting algebras. We prove that every cofinal stable si-logic has the finite model property, and that there are continuum many cofinal stable si-logics that are not stable. We conclude with several examples showing the similarities and differences between the classes of stable, cofinal stable, subframe, and cofinal subframe si-logics.
\end{abstract}

Keywords: Intuitionistic logic, Intuitionistic multi-conclusion consequence relation, Axiomatization, Heyting algebra, Variety, Universal class.

Mathematics Subject Classification: 03B55 - 06D20.

\section{Introduction}

Superintuitionistic logics (si-logics for short) are propositional logics extending the intuitionistic propositional calculus IPC. Consistent si-logics are also known as intermediate logics as they are exactly the logics situated between IPC and the classical propositional calculus CPC. By the Gödel translation, si-logics are closely related to modal logics extending $\mathbf{S 4}$. A detailed account of si-logics and how they relate to modal logics can be found in $[8]$.

Finding uniform axiomatizations for si-logics and modal logics has been a significant problem in the area. First general results in this direction were obtained by Jankov $[19,20]$, de Jongh [10], and Fine [14,15]. They introduced

Presented by Constantine Tsinakis; Received February 21, 2015 
formulas that provide a uniform axiomatization for large classes of si-logics and transitive modal logics. Zakharyaschev $[26,27]$ generalized these results by introducing canonical formulas, which axiomatize all si-logics and all transitive modal logics. Jeřábek [21] further generalized Zakharyaschev's approach by defining canonical multi-conclusion rules that axiomatize all intuitionistic multi-conclusion consequence relations and all transitive modal multi-conclusion consequence relations.

The algebraic counterparts of Zakharyaschev's canonical formulas for silogics are the $(\wedge, \rightarrow)$-canonical formulas of [4]. The $(\wedge, \rightarrow)$-canonical formula of a finite subdirectly irreducible (s.i.) Heyting algebra $A$ fully describes the $\checkmark$-free reduct of $A$, but describes the behavior of the missing connective $\checkmark$ only partially. In fact, the algebraic content of Zakharyaschev's closed domain condition (CDC) is encoded by $D \subseteq A^{2}$, where the behavior of $\vee$ is described fully. The $(\wedge, \rightarrow)$-canonical formulas, though syntactically quite different, serve the same purpose as Zakharyaschev's canonical formulas in providing a uniform axiomatization of all si-logics.

One of the main technical tools in developing the theory of $(\wedge, \rightarrow)$ canonical formulas is Diego's theorem [11] that the variety of implicative meet-semilattices is locally finite. Another locally finite variety closely related to the variety of Heyting algebras is that of bounded distributive lattices. This suggests a different approach to canonical formulas, which was developed in [5], where $(\wedge, \vee)$-canonical formulas were introduced. The $(\wedge, \vee)$-canonical formula of a finite s.i. Heyting algebra $A$ fully describes the bounded lattice reduct of $A$, and only partially the behavior of the missing connective $\rightarrow$. In this case the CDC is encoded by $D \subseteq A^{2}$, where the behavior of $\rightarrow$ is described fully. As in the $(\wedge, \rightarrow)$-case, the $(\wedge, \vee)$-canonical formulas provide a uniform axiomatization of all si-logics.

If $D=A^{2}$, then both $(\wedge, \rightarrow)$ - and $(\wedge, \vee)$-canonical formulas become equivalent to Jankov formulas, and axiomatize splitting si-logics and their joins. If $D=\varnothing$, then $(\wedge, \rightarrow)$-canonical formulas axiomatize subframe and cofinal subframe si-logics, depending on whether or not the behavior of negation is encoded in the formula [4]. If $D=\varnothing$, then $(\wedge, \vee)$-canonical formulas axiomatize a new class of logics, called stable si-logics [5]. Stable si-logics form a well-behaved class of logics having the finite model property (FMP). Many natural si-logics are stable and there is a continuum of stable si-logics.

Stable si-logics can be seen as the $(\wedge, \vee)$-counterpart of subframe silogics. But what is the $(\wedge, \vee)$-counterpart of cofinal subframe si-logics? As 
we pointed out above, for $D=\varnothing$, cofinal subframe si-logics are axiomatized by $(\wedge, \rightarrow, \neg)$-canonical formulas, while subframe si-logics by $(\wedge, \rightarrow)$ canonical formulas. It is only natural to introduce cofinal stable si-logics as the logics axiomatized by $(\wedge, \vee, \neg)$-canonical formulas when $D=\varnothing$. For this we need to work with the pseudocomplemented lattice reduct of Heyting algebras, instead of the bounded lattice reduct like in the case of stable logics. Fortunately, the corresponding variety of pseudocomplemented distributive lattices is locally finite, and hence the algebraic approach is applicable. However, the $(\wedge, \vee, \neg)$-reduct of Heyting algebras is much worse behaved than the $(\wedge, \vee)$-reduct, which results in subtle differences between stable si-logics and cofinal stable si-logics. For example, while stable silogics are axiomatizable by stable formulas, cofinal stable si-logics are not axiomatizable by cofinal stable formulas, instead cofinal stable rules are required.

Because of this, we first generalize $(\wedge, \vee)$-canonical formulas to $(\wedge, \vee)$ canonical rules, which axiomatize all intuitionistic multi-conclusion consequence relations. The $(\wedge, \vee)$-canonical rules are the intuitionistic counterpart of the stable canonical rules of [6], and are an alternative of Jeřábek's canonical multi-conclusion rules [21]. We also indicate how to generalize $(\wedge, \rightarrow)$-canonical formulas to $(\wedge, \rightarrow)$-canonical rules, which provide another uniform axiomatization of all intuitionistic multi-conclusion consequence relations.

Next we generalize stable si-logics to intuitionistic stable multi-conclusion consequence relations, prove that intuitionistic stable multi-conclusion consequence relations have the FMP, and utilize $(\wedge, \vee)$-canonical rules to give a convenient characterization of stable si-logics.

Finally, we generalize $(\wedge, \vee)$-canonical rules to $(\wedge, \vee, \neg)$-canonical rules, which give rise to cofinal stable rules when $D=\varnothing$. We prove that cofinal stable rules axiomatize cofinal stable intuitionistic multi-conclusion consequence relations and cofinal stable si-logics. However, the corresponding formulas axiomatize a larger class of si-logics, properly containing the class of cofinal stable si-logics. A characterization of this class and whether each logic in the class has the FMP remain open problems.

The class of cofinal stable si-logics provides a new class of si-logics. It contains the class of all stable si-logics, and we show that there are continuum many cofinal stable si-logics that are not stable. We conclude the paper with several examples that indicate the similarities and differences between the classes of stable, cofinal stable, subframe, and cofinal subframe si-logics. 


\section{Intuitionistic Multi-conclusion Consequence Relations}

In this section we recall multi-conclusion rules and multi-conclusion consequence relations. For more details see $[18,21]$. A multi-conclusion rule is an expression of the form $\Gamma / \Delta$, where $\Gamma$ and $\Delta$ are finite sets of formulas.

DeFINITION 2.1. An intuitionistic multi-conclusion consequence relation is a set $\mathcal{S}$ of multi-conclusion rules such that

(1) $\varphi / \varphi \in \mathcal{S}$.

(2) $\varphi, \varphi \rightarrow \psi / \psi \in \mathcal{S}$.

(3) $/ \varphi \in \mathcal{S}$ for each theorem $\varphi$ of IPC.

(4) If $\Gamma / \Delta \in \mathcal{S}$, then $\Gamma, \Gamma^{\prime} / \Delta, \Delta^{\prime} \in \mathcal{S}$.

(5) If $\Gamma / \Delta, \varphi \in \mathcal{S}$ and $\Gamma, \varphi / \Delta \in \mathcal{S}$, then $\Gamma / \Delta \in \mathcal{S}$.

(6) If $\Gamma / \Delta \in \mathcal{S}$ and $s$ is a substitution, then $s(\Gamma) / s(\Delta) \in \mathcal{S}$.

We denote the least intuitionistic multi-conclusion consequence relation by $\mathcal{S}_{\text {IPC }}$, and the complete lattice of multi-conclusion consequence relations extending $\mathcal{S}_{\text {IPC }}$ by Ext $\mathcal{S}_{\text {IPC }}$. For a set $\mathcal{R}$ of multi-conclusion rules, let $\mathcal{S}_{\text {IPC }}+\mathcal{R}$ be the least intuitionistic multi-conclusion consequence relation containing $\mathcal{R}$. If $\mathcal{S}=\mathcal{S}_{\text {IPC }}+\mathcal{R}$, then we say that $\mathcal{S}$ is axiomatized by $\mathcal{R}$.

Let ExtIPC be the complete lattice of si-logics. For an intuitionistic multi-conclusion consequence relation $\mathcal{S}$, let $\Lambda(\mathcal{S})=\{\varphi \mid / \varphi \in \mathcal{S}\}$, and for a si-logic $L$, let $\Sigma(L)=\mathcal{S}_{\text {IPC }}+\{/ \varphi \mid \varphi \in L\}$. Then $\Lambda: \operatorname{Ext} \mathcal{S}_{\text {IPC }} \rightarrow$ ExtIPC and $\Sigma:$ ExtIPC $\rightarrow$ Ext $\mathcal{S}_{\text {IPC }}$ are order preserving maps such that $\Lambda(\Sigma(L))=L$ for each $L \in \operatorname{ExtIPC}$ and $\mathcal{S} \supseteq \Sigma(\Lambda(\mathcal{S}))$ for each $\mathcal{S} \in \operatorname{Ext} \mathcal{S}_{\text {IPC }}$. We say that a si-logic $L$ is axiomatized by a set $\mathcal{R}$ of multi-conclusion rules if $L=\Lambda\left(\mathcal{S}_{\text {IPC }}+\mathcal{R}\right)$.

A Heyting algebra $A$ validates a multi-conclusion rule $\Gamma / \Delta$ provided for every valuation $v$ on $A$, if $v(\gamma)=1$ for all $\gamma \in \Gamma$, then $v(\delta)=1$ for some $\delta \in \Delta$. A class $\mathcal{K}$ of Heyting algebras validates $\Gamma / \Delta$ provided each $A \in \mathcal{K}$ validates $\Gamma / \Delta$. If $A$ validates $\Gamma / \Delta$, we write $A=\Gamma / \Delta$, and if $\mathcal{K}$ validates $\Gamma / \Delta$, we write $\mathcal{K} \models \Gamma / \Delta$.

Suppose $\Gamma=\left\{\phi_{1}, \ldots, \phi_{n}\right\}, \Delta=\left\{\psi_{1}, \ldots, \psi_{m}\right\}$, and $\phi_{i}(\bar{x})$ and $\psi_{j}(\bar{x})$ are the terms in the first-order language of Heyting algebras corresponding to the $\phi_{i}$ and $\psi_{j}$. Then $A=\Gamma / \Delta$ iff $A$ is a model of the universal sentence $\forall \bar{x}\left(\bigwedge_{i=1}^{n} \phi_{i}(\bar{x})=1 \rightarrow \bigvee_{j=1}^{m} \psi_{j}(\bar{x})=1\right)$. Conversely, it is easy to see (using the conjunctive normal form) that each universal sentence in the first-order 
language of Heyting algebras can be rewritten as a conjunction of the sentences of the form $\forall \bar{x}\left(\bigwedge_{i=1}^{n} \phi_{i}(\bar{x})=1 \rightarrow \bigvee_{j=1}^{m} \psi_{j}(\bar{x})=1\right)$. Consequently, intuitionistic multi-conclusion consequence relations correspond to universal classes of Heyting algebras (classes of Heyting algebras axiomatized by universal sentences). It is well known (see, e.g., [7, Thm. V.2.20]) that a class of Heyting algebras is a universal class iff it is closed under isomorphisms, subalgebras, and ultraproducts.

On the other hand, si-logics correspond to equationally definable classes of Heyting algebras; that is, models of the sentences $\forall \bar{x} \phi(\bar{x})=1$ in the first-order language of Heyting algebras. It is well known (see, e.g., [7, Thm. II.11.9]) that a class of Heyting algebras is an equational class iff it is a variety (that is, it is closed under homomorphic images, subalgebras, and products).

Let HA be the variety of all Heyting algebras. For an intuitionistic multiconclusion consequence relation $\mathcal{S}$, let $\mathcal{U}(\mathcal{S})=\{A \in \mathrm{HA} \mid A \models \mathcal{S}\}$ be the universal class of Heyting algebras corresponding to $\mathcal{S}$, and for a universal class of Heyting algebras $\mathcal{U}$, let $\mathcal{S}(\mathcal{U})=\mathcal{S}_{\text {IPC }}+\{\Gamma / \Delta \mid \mathcal{U} \models \Gamma / \Delta\}$ be the intuitionistic multi-conclusion consequence relation corresponding to $\mathcal{U}$. Then $\mathcal{S}(\mathcal{U}(\mathcal{S}))=\mathcal{S}$ and $\mathcal{U}(\mathcal{S}(\mathcal{U}))=\mathcal{U}$. This yields an isomorphism between Ext $\mathcal{S}_{\text {IPC }}$ and the complete lattice $\mathrm{U}(\mathrm{HA})$ of universal classes of Heyting algebras (ordered by reverse inclusion).

Similarly, for a si-logic $L$, let $\mathcal{V}(L)=\{A \in \mathrm{HA} \mid A \models L\}$ be the variety of Heyting algebras corresponding to $L$, and for a variety $\mathcal{V}$ of Heyting algebras, let $L(\mathcal{V})=\mathbf{I P C}+\{\varphi \mid \mathcal{V}=\varphi\}$ be the si-logic corresponding to $\mathcal{V}$. Then $L(\mathcal{V}(L))=L$ and $\mathcal{V}(L(\mathcal{V}))=\mathcal{V}$, yielding an isomorphism between ExtIPC and the complete lattice $\mathrm{V}(\mathrm{HA})$ of varieties of Heyting algebras (ordered by reverse inclusion).

Under this correspondence, for an intuitionistic multi-conclusion consequence relation $\mathcal{S}$, the variety $\mathcal{V}(\Lambda(\mathcal{S})$ ) corresponding to the si-logic $\Lambda(\mathcal{S})$ is the variety generated by the universal class $\mathcal{U}(\mathcal{S})$.

\section{3. $(\wedge, \vee)$-Canonical Rules}

We generalize the $(\wedge, \vee)$-canonical formulas of $[5]$ to $(\wedge, \vee)$-canonical rules, and show that each intuitionistic multi-conclusion consequence relation is axiomatizable by $(\wedge, \vee)$-canonical rules. This provides an intuitionistic analogue of similar results in [6]. Our proofs are modifications of those in [6, Sec. 5] and generalize those in [5, Sec. 3]. Therefore, we will only provide their sketches. 
Definition 3.1. Let $A$ be a finite Heyting algebra and let $D \subseteq A^{2}$. We associate with elements of $A$ distinct propositional letters, and define the $(\wedge, \vee)$-canonical rule $\rho(A, D)$ associated with $A$ and $D$ as $\rho(A, D)=\Gamma / \Delta$, where

$$
\begin{aligned}
\Gamma= & \left\{p_{0} \leftrightarrow 0\right\} \cup\left\{p_{1} \leftrightarrow 1\right\} \cup \\
& \left\{p_{a \vee b} \leftrightarrow p_{a} \vee p_{b} \mid a, b \in A\right\} \cup \\
& \left\{p_{a \wedge b} \leftrightarrow p_{a} \wedge p_{b} \mid a, b \in A\right\} \cup \\
& \left\{p_{a \rightarrow b} \leftrightarrow p_{a} \rightarrow p_{b} \mid(a, b) \in D\right\}
\end{aligned}
$$

and

$$
\Delta=\left\{p_{a} \leftrightarrow p_{b} \mid a, b \in A \text { with } a \neq b\right\} .
$$

Proposition 3.2. Let $A$ be a finite Heyting algebra, $D \subseteq A^{2}$, and $B$ be an arbitrary Heyting algebra. Then $B \not \models \rho(A, D)$ iff there is a bounded lattice embedding $h: A \rightarrow B$ such that $h(a \rightarrow b)=h(a) \rightarrow h(b)$ for each $(a, b) \in D$.

Proof. (Sketch) Suppose $B \not \models \rho(A, D)$ and let $v$ be a valuation on $B$ witnessing that $B \not \models \rho(A, D)$. Then $v(\gamma)=1$ for all $\gamma \in \Gamma$ and $v(\delta) \neq 1$ for all $\delta \in \Delta$. It then follows that the map $h: A \rightarrow B$ defined by $h(a)=v\left(p_{a}\right)$ for all $a \in A$ is a bounded lattice embedding such that $h(a \rightarrow b)=h(a) \rightarrow h(b)$ for all $(a, b) \in D$. Conversely, if $h: A \rightarrow B$ is a bounded lattice embedding such that $h(a \rightarrow b)=h(a) \rightarrow h(b)$ for each $(a, b) \in D$, then the valuation $v$ on B defined by $v\left(p_{a}\right)=h(a)$ for all $a \in A$ witnesses that $B \not \models \rho(A, D)$.

Proposition 3.3. If $\mathcal{S}_{\text {IPC }} \forall \Gamma / \Delta$, then there are finite Heyting algebras $A_{1}, \ldots, A_{m}$ and $D_{1}, \ldots, D_{m}$ with $D_{i} \subseteq A_{i}^{2}$ for $1 \leq i \leq m$, such that for each Heyting algebra $B$, we have $B \forall=\Gamma / \Delta$ iff there is $1 \leq i \leq m$ and a bounded lattice embedding $h: A_{i} \rightarrow B$ with $h(a \rightarrow b)=h(a) \rightarrow h(b)$ for all $(a, b) \in D_{i}$.

Proof. (Sketch) Let $\Xi$ be the set of all subformulas of formulas in $\Gamma \cup \Delta$. Then $\Xi$ is finite. Let $n$ be the cardinality of $\Xi$. Since the variety of bounded distributive lattices is locally finite, up to isomorphism, there are only finitely many pairs $(A, D)$ satisfying the following two conditions:

(i) $A$ is a finite Heyting algebra that is at most $n$-generated as a bounded distributive lattice and $A \not \models \Gamma / \Delta$.

(ii) $D=\{(v(\varphi), v(\psi)) \mid \varphi \rightarrow \psi \in \Xi\}$, where $v$ is a valuation on $A$ witnessing $A \not \forall \Gamma / \Delta$.

If $\left(A_{1}, D_{1}\right), \ldots,\left(A_{m}, D_{m}\right)$ is their enumeration, then we show that this collection is as required. Suppose $B$ is a Heyting algebra with $B \not \models \Gamma / \Delta$. If $v$ is a valuation witnessing that $B \not \models \Gamma / \Delta$, then let $A$ be the bounded sublattice 
of $B$ generated by the set $v[\Xi]$, and let $D=\{(v(\varphi), v(\psi)) \mid \varphi \rightarrow \psi \in \Xi\}$. Then $(A, D)$ is up to isomorphism one of $\left(A_{i}, D_{i}\right)$ and the inclusion from $A$ to $B$ gives rise to a bounded lattice embedding $h: A_{i} \rightarrow B$ such that $h(a \rightarrow b)=h(a) \rightarrow h(b)$ for all $(a, b) \in D_{i}$. Conversely, let $h: A_{i} \rightarrow B$ be a bounded lattice embedding with $h(a \rightarrow b)=h(a) \rightarrow h(b)$ for all $(a, b) \in D_{i}$. Let $v$ be the valuation on $A_{i}$ that gave rise to $D_{i}=\{(v(\varphi), v(\psi)) \mid \varphi \rightarrow \psi \in$ $\Xi\}$. Then $h \circ v$ is a valuation on $B$ witnessing that $B \not \models \Gamma / \Delta$.

Proposition 3.4. Every intuitionistic multi-conclusion consequence relation $\mathcal{S}$ is axiomatizable by $(\wedge, \vee)$-canonical rules. Moreover, if $\mathcal{S}$ is finitely axiomatizable, then it is axiomatizable by finitely many $(\wedge, \vee)$-canonical rules.

Proof. (Sketch) Suppose $\mathcal{S}=\mathcal{S}_{\text {IPC }}+\left\{\Gamma_{i} / \Delta_{i} \mid i \in I\right\}$ with $\Gamma_{i} / \Delta_{i} \notin \mathcal{S}_{\text {IPC }}$. For every $\Gamma_{i} / \Delta_{i}$ pick $\left(A_{i_{1}}, D_{i_{1}}\right), \ldots,\left(A_{i_{m_{i}}}, D_{i_{m_{i}}}\right)$ according to Proposition 3.3. Then $\mathcal{S}=\mathcal{S}_{\text {IPC }}+\left\{\rho\left(A_{i_{j}}, D_{i_{j}}\right) \mid i \in I, 1 \leq j \leq m_{i}\right\}$.

REMARK 3.5. The $(\wedge, \rightarrow)$-canonical formulas of [4] can also be generalized to $(\wedge, \rightarrow)$-canonical rules. This yields a slightly different axiomatization of all intuitionistic multi-conclusion consequence relations. Namely, let $A$ be a finite Heyting algebra and let $D \subseteq A^{2}$. We associate with elements of $A$ distinct propositional letters, and define the $(\wedge, \rightarrow, 0)$-canonical rule $\zeta(A, D)$ associated with $A$ and $D$ as $\zeta(A, D)=\Gamma / \Delta$, where

$$
\begin{aligned}
\Gamma= & \left\{p_{a \wedge b} \leftrightarrow p_{a} \wedge p_{b} \mid a, b \in A\right\} \cup \\
& \left\{p_{a \rightarrow b} \leftrightarrow p_{a} \rightarrow p_{b} \mid a, b \in A\right\} \cup \\
& \left\{p_{\neg a} \leftrightarrow \neg p_{a} \mid a \in A\right\} \cup \\
& \left\{p_{a \vee b} \leftrightarrow p_{a} \vee p_{b} \mid(a, b) \in D\right\}
\end{aligned}
$$

and

$$
\Delta=\left\{p_{a} \leftrightarrow p_{b} \mid a, b \in A \text { with } a \neq b\right\} .
$$

Then the results of [4] can be generalized to prove that each intuitionistic multi-conclusion consequence relation $\mathcal{S}$ is axiomatizable by $(\wedge, \rightarrow, 0)$ canonical rules. Moreover, if $\mathcal{S}$ is finitely axiomatizable, then it is axiomatizable by finitely many $(\wedge, \rightarrow, 0)$-canonical rules. In fact, the same way $(\wedge, \rightarrow)$-canonical formulas are an algebraic counterpart of Zakharyaschev's canonical formulas for si-logics, $(\wedge, \rightarrow)$-canonical rules are an algebraic counterpart of Jeřábek's canonical rules for intuitionistic multi-conclusion consequence relations.

REMARK 3.6. In [9] Citkin defines characteristic rules for finite partial Heyting algebras and shows that every intuitionistic multi-conclusion consequence relation is axiomatizable by these rules. Citkin's rules are similar to 
stable canonical rules. The essential difference between the two approaches is that Citkin's rules are defined for finite partial algebras, whereas stable canonical rules are defined for finite total algebras. A partial algebra is an algebra, where the operations are defined only partially. By [9, Thm. 4.1], a Heyting algebra $B$ refutes the characteristic rule of a finite partial Heyting algebra $A$ iff there is a partial subalgebra $C$ of $B$ isomorphic to $A$. In this paper all the algebras and embeddings we consider are total.

\section{Stable Multi-conclusion Consequence Relations}

Let $A$ be a finite Heyting algebra and let $D \subseteq A^{2}$. Then there are two obvious extreme cases, when $D=A^{2}$ and when $D=\varnothing$. If $D=A^{2}$, then we call the $(\wedge, \vee)$-canonical rule $\rho(A, D)$ a Jankov rule. As follows from [21, Sec. 6] (see also [6, Sec. 7]), Jankov rules axiomatize splittings and join splittings in the lattice of all intuitionistic multi-conclusion consequence relations.

If $D=\varnothing$, then we call $\rho(A, D)$ a stable rule and denote it by $\rho(A)$. In this section we show that stable rules axiomatize stable multi-conclusion consequence relations, thus generalizing the results of [5, Sec. 6], and providing intuitionistic analogues of the results of $[6$, Sec. 7].

Definition 4.1. (1) We call a class $\mathcal{K}$ of Heyting algebras stable provided for all Heyting algebras $A, B$, if $A$ is isomorphic to a bounded sublattice of $B$ and $B \in \mathcal{K}$, then $A \in \mathcal{K}$.

(2) We call an intuitionistic multi-conclusion consequence relation $\mathcal{S}$ stable provided its corresponding universal class $\mathcal{U}(\mathcal{S})$ is stable.

The next proposition is immediate from the previous section.

Proposition 4.2. Let $A$ be a finite Heyting algebra. For every Heyting algebra $B$, we have $B \not \models \rho(A)$ iff there is a bounded lattice embedding $h$ : $A \rightarrow B$.

Suppose $B$ is a Heyting algebra, $v$ is a valuation on $B$, and $\Gamma$ is a set of formulas. We write $v(\Gamma)=1$ if $v(\gamma)=1$ for all $\gamma \in \Gamma$, and $v(\Gamma) \neq 1$ if $v(\gamma) \neq 1$ for all $\gamma \in \Gamma$. The next lemma generalizes [5, Lem. 3.6].

Lemma 4.3. If a Heyting algebra $B$ refutes a multi-conclusion rule $\Gamma / \Delta$, then there is a finite Heyting algebra $A$ such that $A$ is a bounded sublattice of $B$ and $A$ refutes $\Gamma / \Delta$.

Proof. Since $B \not \models \Gamma / \Delta$, there is a valuation $v_{B}$ on $B$ such that $v_{B}(\Gamma)=1$ and $v_{B}(\Delta) \neq 1$. Let $\Xi$ be the set of subformulas of formulas in $\Gamma \cup \Delta$, and 
let $A$ be the bounded sublattice of $B$ generated by $v_{B}[\Xi]$. Since $\Xi$ is finite and bounded distributive lattices are locally finite, we see that $A$ is finite. Therefore, $A$ is a finite Heyting algebra, where

$$
a \rightarrow_{A} b=\bigvee\{x \in A \mid a \wedge x \leq b\}
$$

for all $a, b \in A$. It is then easy to see that for all $a, b \in A$, we have $a \rightarrow_{A} b \leq$ $a \rightarrow_{B} b$ and $a \rightarrow_{A} b=a \rightarrow_{B} b$ provided $a \rightarrow_{B} b \in A$. Define a valuation $v_{A}$ on $A$ by $v_{A}(p)=v_{B}(p)$ for $p \in \Xi$ and $v_{A}(p)$ is an arbitrary element of $A$ otherwise. It is straightforward to see that $v_{A}(\varphi)=v_{B}(\varphi)$ for all $\varphi \in \Xi$. Thus, $v_{A}(\Gamma)=1_{A}$ and $v_{A}(\Delta) \neq 1_{A}$, and hence $A \not \models \Gamma / \Delta$.

As an immediate corollary, we obtain that stable multi-conclusion consequence relations have the FMP.

Proposition 4.4. Every stable multi-conclusion consequence relation has the FMP.

Proof. Suppose $\mathcal{S}$ is a stable multi-conclusion consequence relation and $\mathcal{S} \nvdash \Gamma / \Delta$. Then there is $B \in \mathcal{U}(\mathcal{S})$ with $B \not \models \Gamma / \Delta$. By Lemma 4.3, there is a bounded lattice embedding $A \hookrightarrow B$ with $A$ finite and $A \not \models \Gamma / \Delta$. Since $\mathcal{U}(\mathcal{S})$ is stable, $A \in \mathcal{U}(\mathcal{S})$. Thus, $\mathcal{S}$ has the FMP.

We conclude this section by proving that stable multi-conclusion consequence relations are exactly the multi-conclusion consequence relations that are axiomatizable by stable rules. This generalizes [5, Thm. 6.11] and is an intuitionistic analogue of [6, Thm. 7.3].

Proposition 4.5. An intuitionistic multi-conclusion consequence relation $\mathcal{S}$ is stable iff it is axiomatizable by stable rules.

Proof. First suppose that $\mathcal{S}$ is stable. We show that $\mathcal{S}=\mathcal{S}_{\text {IPC }}+\{\rho(A) \mid$ $A$ finite, $A \notin \mathcal{U}(\mathcal{S})\}$. Let $B \in \mathrm{HA}$. Suppose $B \models \mathcal{S}$. If there is a finite $A \notin \mathcal{U}(\mathcal{S})$ such that $B \not \models \rho(A)$, then by Proposition 4.2 , there is a bounded lattice embedding $A \hookrightarrow B$. Since $\mathcal{U}(\mathcal{S})$ is stable, $A \in \mathcal{U}(\mathcal{S})$, a contradiction. Therefore, $B \models\{\rho(A) \mid A$ finite, $A \notin \mathcal{U}(\mathcal{S})\}$. Conversely, if $B \not \models \mathcal{S}$, then there is a rule $\Gamma / \Delta$ in $\mathcal{S}$ such that $B \not \models \Gamma / \Delta$. By Lemma 4.3 , there is a finite Heyting algebra $A$ such that $A$ is a bounded sublattice of $B$ and $A \not \models \Gamma / \Delta$. Therefore, $A \not \models \mathcal{S}$. By Proposition 4.2, $B \not \models \rho(A)$. Thus, $B \not \models$ $\mathcal{S}_{\text {IPC }}+\{\rho(A) \mid A$ finite, $A \notin \mathcal{U}(\mathcal{S})\}$.

Next suppose that $\mathcal{S}=\mathcal{S}_{\text {IPC }}+\left\{\rho\left(A_{i}\right) \mid i \in I\right\}$. Let $A, B$ be Heyting algebras such that $A$ is isomorphic to a bounded sublattice of $B$ and $B \in \mathcal{U}(\mathcal{S})$. If $A \notin \mathcal{U}(\mathcal{S})$, then there is $i \in I$ such that $A \not \forall \rho\left(A_{i}\right)$. By Proposition 4.2, there is a bounded lattice embedding $A_{i} \hookrightarrow A$. Therefore, 
there is a bounded lattice embedding $A_{i} \hookrightarrow B$, so applying Proposition 4.2 again yields $B \not \models \rho\left(A_{i}\right)$. Thus, $B \notin \mathcal{U}(\mathcal{S})$, a contradiction. This shows that $A \in \mathcal{U}(\mathcal{S})$, so $\mathcal{U}(\mathcal{S})$ is a stable class, and hence $\mathcal{S}$ is stable.

\section{Stable Logics}

In the previous section we saw that stable multi-conclusion consequence relations provide a generalization of stable si-logics introduced in [5]. As we will see now, stable multi-conclusion consequence relations also aid in obtaining a number of convenient characterizations of stable si-logics.

Recall from [5] that for a finite s.i. Heyting algebra $A$ and $D \subseteq A^{2}$, the $(\wedge, \vee)$-canonical formula associated with $A$ and $D$ is $\gamma(A, D)=\wedge \Gamma \rightarrow \bigvee \Delta$. If $D=\varnothing$, then $\gamma(A, D)$ is denoted by $\gamma(A)$, and is called the stable formula associated with $A$.

A si-logic $L$ is stable provided for arbitrary s.i. Heyting algebras $A, B$, if $A$ is isomorphic to a bounded sublattice of $B$ and $B \models L$, then $A \models L$. By [5, Sec. 6], stable si-logic have the FMP, and a si-logic $L$ is stable iff it is axiomatizable by stable formulas.

In [5, Thm. 6.3] it is proved that if $A, B$ are s.i. Heyting algebras with $A$ finite, then $B \not \models \gamma(A)$ iff $A$ is isomorphic to a bounded sublattice of $B$. We show that in the theorem the condition that $B$ is s.i. can be weakened to $B$ being well-connected, where we recall that $B$ is well-connected provided $a \vee b=1 \Rightarrow a=1$ or $b=1$. In other words, $B$ is well-connected iff $B$ is finitely subdirectly irreducible.

Proposition 5.1. Let $A, B$ be Heyting algebras with $A$ finite and s.i. and $B$ well-connected. Then $B \not \models \gamma(A)$ iff $A$ is isomorphic to a bounded sublattice of $B$.

Proof. First suppose that $A$ is isomorphic to a bounded sublattice of $B$. The same argument as in the proof of [5, Thm. 6.3] shows that there is a valuation $v$ on $B$ refuting $\gamma(A)$ so that $v(\gamma)=1_{B}$ for each $\gamma \in \Gamma$ and $v(\delta) \neq 1_{B}$ for each $\delta \in \Delta$. Therefore, since $B$ is well-connected and $\Gamma, \Delta$ are finite, $v(\bigwedge \Gamma)=\bigwedge\{v(\gamma) \mid \gamma \in \Gamma\}=1_{B}$ and $v(\bigvee \Delta)=\bigvee\{v(\delta) \mid \delta \in \Delta\} \neq 1_{B}$. Thus, $B \not \models \gamma(A)$.

For the converse, we prove a stronger statement, that for an arbitrary $B$, from $B \forall \gamma(A)$ it follows that $A$ is isomorphic to a bounded sublattice of $B$.

Claim 5.2. Let $A, B$ be Heyting algebras with $A$ finite and s.i. If $B \not \models \gamma(A)$, then $A$ is isomorphic to a bounded sublattice of $B$. 
Proof of Claim. If $B \not \models \gamma(A)$, then by [5, Lem. 3.6], there is a finite Heyting algebra $B^{\prime}$ such that $B^{\prime}$ is a bounded sublattice of $B$ and $B^{\prime} \not \models \gamma(A)$. By [5, Thm. 3.4], there is a homomorphic image $C$ of $B^{\prime}$ and a bounded lattice embedding $f: A \rightarrow C$. Since $B^{\prime}$ is finite, applying [5, Lem. 6.2] yields a bounded lattice embedding $f^{\prime}: A \rightarrow B^{\prime}$. Thus, $A$ is isomorphic to a bounded sublattice of $B$.

This completes the proof of the proposition.

We are ready to prove the main result of the section. For a variety $\mathcal{V}$ of Heyting algebras, let $\mathcal{V}_{w c}$ be the class of well-connected members of $\mathcal{V}$.

TheOREM 5.3. For a si-logic $L$ and the corresponding variety $\mathcal{V}$ of Heyting algebras, the following are equivalent:

(1) $L$ is stable.

(2) $L$ is axiomatizable by stable formulas.

(3) $L$ is axiomatizable by stable rules.

(4) $\mathcal{V}_{w c}$ is stable.

$(5) \mathcal{V}$ is generated by a stable universal class.

(6) $\mathcal{V}$ is generated by a stable class.

Proof. (1) $\Leftrightarrow(2)$ : This is proved in [5, Thm. 6.11].

$(2) \Rightarrow(4)$ : By $(2)$, there is a family $\left\{A_{i} \mid i \in I\right\}$ of finite s.i. Heyting algebras such that $L=\mathbf{I P C}+\left\{\gamma\left(A_{i}\right) \mid i \in I\right\}$. Let $A, B$ be Heyting algebras with $B \in \mathcal{V}_{w c}$ and $A$ isomorphic to a bounded sublattice of $B$. Since bounded sublattices preserve well-connectedness, we see that $A$ is also well-connected. Therefore, it remains to prove that $A \in \mathcal{V}$. If not, then there is $i \in I$ such that $A \not \models \gamma\left(A_{i}\right)$. By Proposition 5.1, $A_{i}$ is isomorphic to a bounded sublattice of $A$. By transitivity, $A_{i}$ is isomorphic to a bounded sublattice of $B$. Since $B$ is well-connected, applying Proposition 5.1 again yields $B \not \forall \gamma\left(A_{i}\right)$, a contradiction. Thus, $A \in \mathcal{V}_{w c}$.

$(4) \Rightarrow(5)$ : The class $\mathcal{V}_{w c}$ is universal since it is definable over $\mathcal{V}$ by the universal formula $\forall x y(x \vee y=1 \rightarrow(x=1 \vee y=1))$. By (4), $\mathcal{V}_{w c}$ is stable. Since $\mathcal{V}_{w c}$ contains all s.i. members of $\mathcal{V}$, we see that $\mathcal{V}_{w c}$ generates $\mathcal{V}$.

$(5) \Rightarrow(6)$ : This is obvious.

$(6) \Rightarrow(2)$ : Suppose that $\mathcal{V}$ is generated by a stable class $\mathcal{K}$. First we show that $L$ has the FMP.

Claim 5.4. If $\varphi \notin L$, then there is a finite $A \in \mathcal{K}$ with $A \not \models \varphi$. In particular, $L$ has the FMP. 
Proof of Claim. If $\varphi \notin L$, then there is $B \in \mathcal{K}$ with $B \not \models \varphi$. By [5, Lem. 3.6], there is a finite Heyting algebra $A$ such that $A$ is a bounded sublattice of $B$ and $A \not \models \varphi$. Since $\mathcal{K}$ is stable, $A \in \mathcal{K}$. Thus, $L$ has the FMP.

Let $L^{\prime}=\mathbf{I P C}+\{\gamma(A) \mid A$ is finite, s.i., and $A \notin \mathcal{V}\}$. We claim that $L=L^{\prime}$. By Claim 5.4, $L$ has the FMP, and by [5, Sec. 6], $L^{\prime}$ has the FMP. Therefore, it is sufficient to show that for each finite s.i. Heyting algebra $B$, we have $B \models L$ iff $B \models L^{\prime}$. Suppose $B$ is a finite s.i. Heyting algebra. If $B \not L^{\prime}$, then there is a finite s.i. Heyting algebra $A \notin \mathcal{V}$ such that $B \not \models \gamma(A)$.

Claim 5.5. $\gamma(A) \in L$.

Proof of Claim. If $\gamma(A) \notin L$, then since $\mathcal{V}$ is generated by $\mathcal{K}$, there is $C \in \mathcal{K}$ with $C \not \forall \gamma(A)$. By Claim 5.2, $A$ is isomorphic to a bounded sublattice of $C$. Since $\mathcal{K}$ is stable, $A \in \mathcal{K}$, which contradicts $A \notin \mathcal{V}$. Thus, $\gamma(A) \in L$.

By Claim 5.5, $\gamma(A) \in L$. Therefore, $B \not \forall L$, showing that $L^{\prime} \subseteq L$. Conversely, suppose that $B \not \models L$. By definition, $\gamma(B) \in L^{\prime}$. Since $B \not \models \gamma(B)$, it follows that $B \not \forall L^{\prime}$. This shows that $L \subseteq L^{\prime}$. Thus, $L=L^{\prime}$, which yields that $L$ is axiomatizable by stable formulas.

$(3) \Rightarrow(5)$ : Suppose $L$ is axiomatizable by stable rules. Then there is a family $\left\{A_{i} \mid i \in I\right\}$ of finite Heyting algebras such that $L=\Lambda(\mathcal{S})$, where $\mathcal{S}=\mathcal{S}_{\text {IPC }}+\left\{\rho\left(A_{i}\right) \mid i \in I\right\}$. Therefore, $\mathcal{V}$ is generated by $\mathcal{U}(\mathcal{S})$, which by Proposition 4.5 is a stable universal class.

$(5) \Rightarrow(3)$ : Suppose $\mathcal{V}$ is generated by a stable universal class $\mathcal{U}$. Let $\mathcal{S}$ be the intuitionistic multi-conclusion consequence relation corresponding to $\mathcal{U}$. By Proposition 4.5, $\mathcal{S}=\mathcal{S}_{\text {IPC }}+\{\rho(A) \mid A$ finite, $A \notin \mathcal{U}\}$. Therefore, since $\mathcal{V}$ is generated by $\mathcal{U}$, we see that $L=\Lambda(\mathcal{S})$. Thus, $L$ is axiomatizable by stable rules.

REMARK 5.6. Let $L$ be a si-logic and let $\mathcal{V}$ be the corresponding variety of Heyting algebras. By Theorem 5.3, $L$ is stable iff $\mathcal{V}$ is generated by a stable class. This, however, does not mean that $\mathcal{V}$ itself is a stable class. In fact, the variety of all Heyting algebras is the only nontrivial stable variety of Heyting algebras. This can be seen as follows. Suppose $\mathcal{V}$ is a nontrivial stable variety of Heyting algebras. Since $\mathcal{V}$ is nontrivial, it contains the variety of Boolean algebras. Let $A$ be an arbitrary Heyting algebra, and let $\mathcal{B}(A)$ be the free Boolean extension of $A$ (see, e.g., [1, Ch. V.4]). Then $\mathcal{B}(A)$ is a Boolean algebra and $A$ is isomorphic to a bounded sublattice of $\mathcal{B}(A)$. Therefore, $\mathcal{B}(A) \in \mathcal{V}$, and since $\mathcal{V}$ is stable, $A \in \mathcal{V}$. Thus, $\mathcal{V}$ is the variety of all Heyting algebras. 
REMARK 5.7. Let $\left\{A_{i} \mid i \in I\right\}$ be a family of finite s.i. Heyting algebras. Then IPC $+\left\{\gamma\left(A_{i}\right) \mid i \in A\right\}=\Lambda\left(\mathcal{S}_{\text {IPC }}+\left\{\rho\left(A_{i}\right) \mid i \in I\right\}\right)$; that is, the silogic axiomatized by the stable formulas $\left\{\gamma\left(A_{i}\right) \mid i \in I\right\}$ is the same as the si-logic axiomatized by the corresponding stable rules $\left\{\rho\left(A_{i}\right) \mid i \in I\right\}$. This can be seen as follows. By [5, Thm. 6.3] and Proposition 4.2, for every s.i. Heyting algebra $B$, we have $B \models \gamma\left(A_{i}\right)$ iff $B \models \rho\left(A_{i}\right)$. Therefore, the universal class $\mathcal{U}$ corresponding to the intuitionistic multi-conclusion consequence relation $\mathcal{S}_{\text {IPC }}+\left\{\rho\left(A_{i}\right) \mid i \in I\right\}$ and the variety $\mathcal{V}$ corresponding to the si-logic IPC $+\left\{\gamma\left(A_{i}\right) \mid i \in A\right\}$ contain the same s.i. members. Thus, $\mathcal{V}$ is generated by $\mathcal{U}$, yielding the result. As we will see in Remark 7.14, this does not generalize to a similar statement about $(\wedge, \vee)$-canonical formulas and $(\wedge, \vee)$-canonical rules.

\section{Cofinal Stable Multi-conclusion Consequence Relations}

While the theories of $(\wedge, \vee)$ - and $(\wedge, \rightarrow)$-canonical rules and formulas have many similarities, there is one important difference. Namely, in the $(\wedge, \rightarrow)$ case, since $\rightarrow$ is preserved, preserving 0 implies preserving $\neg$. This is not true in the $(\wedge, \vee)$-case. Therefore, in the $(\wedge, \rightarrow)$-case, when 0 is preserved, putting $D=\varnothing$ yields cofinal subframe rules and formulas. To obtain subframe rules and formulas, along with $D=\varnothing$, we also have to take out $\left\{p_{\neg a} \leftrightarrow \neg p_{a} \mid a \in A\right\}$ from $\Gamma$. Since in the $(\wedge, \vee)$-case $\left\{p_{\neg a} \leftrightarrow \neg p_{a} \mid a \in A\right\}$ is not part of $\Gamma$, by taking $D=\varnothing$ we only obtain stable rules and formulas, which are analogues of subframe rules and formulas. In order to obtain analogues of cofinal subframe rules and formulas, we have to add $\left\{p_{\neg a} \leftrightarrow \neg p_{a} \mid a \in A\right\}$ to $\Gamma$. Thus, instead of working with bounded distributive sublattices of Heyting algebras, we will have to work with pseudocomplemented sublattices. This is what we do next.

We recall that a pseudocomplemented distributive lattice is a bounded distributive lattice $L$ with an additional unary operation $\neg: L \rightarrow L$ satisfying $a \wedge b=0$ iff $a \leq \neg b$ (see, e.g., [1, Ch. VIII]). Let PDLat be the class of pseudocomplemented distributive lattices. Clearly PDLat is contained in the class DLat of bounded distributive lattices and contains the class HA of Heyting algebras.

It is well known that PDLat is a variety. We will require that PDLat is a locally finite variety. Since we were unable to find a proof of this result in the literature, we give a proof of it below.

TheOREM 6.1. PDLat is locally finite. 
Proof. We utilize the criterion of local finiteness from [2, Thm. 3.7] stating that a variety $\mathcal{V}$ of finite signature is locally finite iff for each $n \in \mathbb{N}$ there is $m(n) \in \mathbb{N}$ such that the cardinality of all $n$-generated s.i. members of $\mathcal{V}$ is bounded above by $m(n)$. It is well known (see, e.g., [1, Thm. VIII.5.1]) that s.i. members of PDLat are of the form $B \oplus 1$, where $B$ is a Boolean algebra and $-\oplus 1$ is the operation of adjoining a new top. We claim that the cardinality of each $n$-generated s.i. $A \in$ PDLat is bounded above by $m(n)=2^{2^{n}}+1$. Indeed, suppose $A \cong B \oplus 1$ is generated by $g_{1}, \ldots, g_{n}$. Without loss of generality we may assume that $g_{1}, \ldots, g_{n} \in B$. Therefore, $g_{1}, \ldots, g_{n}$ generate $B$ as a Boolean algebra. Thus, the cardinality of $B$ is bounded above by $2^{2^{n}}$. This yields that the cardinality of $A$ is bounded above by $2^{2^{n}}+1$. Consequently, the criterion applies.

We are ready to define cofinal stable classes of Heyting algebras and cofinal stable rules.

Definition 6.2. (1) We call a class $\mathcal{K}$ of Heyting algebras cofinal stable provided for Heyting algebras $A, B$, if $A$ is isomorphic to a pseudocomplemented sublattice of $B$ and $B \in \mathcal{K}$, then $A \in \mathcal{K}$.

(2) Let $A$ be a finite Heyting algebra. The cofinal stable rule associated with $A$ is the rule $\sigma(A)=\Gamma / \Delta$, where

$$
\begin{aligned}
\Gamma= & \left\{p_{0} \leftrightarrow 0\right\} \cup \\
& \left\{p_{a \vee b} \leftrightarrow p_{a} \vee p_{b} \mid a, b \in A\right\} \cup \\
& \left\{p_{a \wedge b} \leftrightarrow p_{a} \wedge p_{b} \mid a, b \in A\right\} \cup \\
& \left\{p_{\neg a} \leftrightarrow \neg p_{a} \mid a \in A\right\}
\end{aligned}
$$

and

$$
\Delta=\left\{p_{a} \leftrightarrow p_{b} \mid a, b \in A \text { with } a \neq b\right\} .
$$

REMARK 6.3. There is no need to add $p_{1} \leftrightarrow 1$ to $\Gamma$ because $p_{\neg 0} \leftrightarrow \neg p_{0}$ and $p_{0} \leftrightarrow 0$ are contained in $\Gamma$.

Using that PDLat is locally finite, the statements and proofs of Section 4 generalize to the setting of cofinal stable rules. Namely, Proposition 4.2 generalizes to the following proposition.

Proposition 6.4. Suppose $A, B$ are Heyting algebras with $A$ finite. Then $B \not \models \sigma(A)$ iff $A$ is isomorphic to a pseudocomplemented sublattice of $B$.

Also, Proposition 4.5 generalizes to the following proposition.

Proposition 6.5. An intuitionistic multi-conclusion consequence relation $\mathcal{S}$ is axiomatizable by cofinal stable rules iff the corresponding universal class $\mathcal{U}(\mathcal{S})$ of Heyting algebras is cofinal stable. 
We omit the proofs since they are virtually the same as the relevant proofs in Section 4, but use local finiteness of PDLat instead of local finiteness of DLat.

REMARK 6.6. Not only stable rules have an obvious generalization to cofinal stable rules, but also $(\wedge, \vee)$-canonical rules have an obvious generalization to $(\wedge, \vee, \neg)$-canonical rules. Suppose $A$ is a finite Heyting algebra and $D \subseteq A^{2}$. We associate with elements of $A$ distinct propositional letters, and define the $(\wedge, \vee, \neg)$-canonical rule $\sigma(A, D)$ associated with $A$ and $D$ as $\sigma(A, D)=\Gamma / \Delta$, where

$$
\begin{aligned}
\Gamma= & \left\{p_{0} \leftrightarrow 0\right\} \cup \\
& \left\{p_{a \wedge b} \leftrightarrow p_{a} \wedge p_{b} \mid a, b \in A\right\} \cup \\
& \left\{p_{a \vee b} \leftrightarrow p_{a} \vee p_{b} \mid a, b \in A\right\} \cup \\
& \left\{p_{\neg a} \leftrightarrow \neg p_{a} \mid a \in A\right\} \cup \\
& \left\{p_{a \rightarrow b} \leftrightarrow p_{a} \rightarrow p_{b} \mid(a, b) \in D\right\}
\end{aligned}
$$

and

$$
\Delta=\left\{p_{a} \leftrightarrow p_{b} \mid a, b \in A \text { with } a \neq b\right\} .
$$

All proofs from Section 3 have an obvious translation to the $(\wedge, \vee, \neg)$-setting by utilizing local finiteness of the variety of pseudocomplemented distributive lattices instead of the variety of bounded distributive lattices. Consequently, $(\wedge, \vee, \neg)$-canonical rules provide yet another uniform axiomatization of all intuitionistic multi-conclusion consequence relations.

\section{Cofinal Stable Logics}

The same way we generalized stable rules to cofinal stable rules, we can generalize stable formulas to cofinal stable formulas. However, unlike the stable case, it is no longer true that cofinal stable logics are axiomatizable by cofinal stable formulas. This mismatch is essentially due to the fact that the analogue of [5, Lem. 6.2] fails for the $(\wedge, \vee, \neg)$-reducts of Heyting algebras. Nevertheless, we will show that cofinal stable logics are axiomatizable by cofinal stable rules.

Let $A$ be a finite s.i. Heyting algebra, and let $\sigma(A)=\Gamma / \Delta$ be the cofinal stable rule associated with $A$. We define the cofinal stable formula associated with $A$ as $\delta(A)=\bigwedge \Gamma \rightarrow \bigvee \Delta$. Then [5, Thm. 3.4] has an obvious generalization to the following proposition.

Proposition 7.1. Let $A, B$ be Heyting algebras with $A$ finite and s.i. Then $B \not \models \delta(A)$ iff there is a s.i. homomorphic image $C$ of $B$ such that $A$ is isomorphic to a pseudocomplemented sublattice of $C$. 
If $B$ is well-connected and we work with $\gamma(A)$ instead of $\delta(A)$, then as is shown in Proposition 5.1, the homomorphic image $C$ can be omitted from the consideration. We will see that this is no longer true if we work with $\delta(A)$. However, one implication of the proposition remains true, with the same proof.

Lemma 7.2. Let $A, B$ be Heyting algebras with $A$ finite and s.i. and $B$ wellconnected. If $A$ is isomorphic to a pseudocomplemented sublattice of $B$, then $B \not \models \delta(A)$.

To see that the converse of Lemma 7.2 does not hold, we recall the representation theorem for pseudocomplemented distributive lattices. Since pseudocomplemented distributive lattices are situated between bounded distributive lattices and Heyting algebras, their representation is closely related to the Priestley representation of bounded distributive lattices and the Esakia representation of Heyting algebras.

We call a subset $U$ of a partially ordered set $X$ an upset if $x \in U$ and $x \leq y$ imply $y \in U$; we define downsets dually. For $U \subseteq X$, let $\uparrow U$ be the upset and $\downarrow U$ be the downset generated by $U$. If $U=\{x\}$, then we write $\uparrow x$ and $\downarrow x$, respectively. A Priestley space is a compact ordered space $X$ such that $x \not \leq y$ implies that there is a clopen (closed and open) upset of $X$ containing $x$ and missing $y$. A map between Priestley spaces is a Priestley morphism if it is continuous and order preserving. By Priestley duality [23,24], the category DLat of bounded distributive lattices and bounded lattice homomorphisms is dually equivalent to the category Pries of Priestley spaces and Priestley morphisms.

An Esakia space is a Priestley space in which $\downarrow U$ is open for each open set $U$. A map $f: X \rightarrow Y$ between Esakia spaces is an Esakia morphism if it is a continuous p-morphism, where we recall that $f$ is a p-morphism provided $f(\uparrow x)=\uparrow f(x)$. By Esakia duality [12], the category HA of Heyting algebras and Heyting homomorphisms is dually equivalent to the category Esa of Esakia spaces and Esakia morphisms.

Since pseudocomplemented distributive lattices are situated between Heyting algebras and bounded distributive lattices, dual spaces of pseudocomplemented distributive lattices are situated between Esakia spaces and Priestley spaces. They were characterized by Priestley in [25]. For a subset $U$ of a partially ordered set $X$, let $\max (U)$ be the set of maximal points of $U(m \in \max (U)$ provided $(\forall y \in U)(x \leq y \Rightarrow x=y))$. We call a Priestley space $X$ a $P C$-space if $\downarrow U$ is open for each open upset $U$. Clearly each Esakia space is a PC-space, but the converse is not true in general. We call a map $f: X \rightarrow Y$ between ordered spaces a $q$-morphism 
if $\max \uparrow f(x)=f(\max \uparrow x)$. By [25], the category PDLat of pseudocomplemented distributive lattices and pseudocomplemented lattice homomorphisms is dually equivalent to the category PCS of PC-spaces and continuous q-morphisms.

The next lemma gives several characterizations of q-morphisms.

Lemma 7.3. Let $f: X \rightarrow Y$ be a Priestley morphism between $P C$-spaces. The following are equivalent.

(1) $f$ is a q-morphism.

(2) For all $x \in X$, we have $\max \uparrow f(x) \subseteq f(\max \uparrow x)$.

(3) For all $x \in X$ and $y \in Y$, from $f(x) \leq y$ it follows that there is $x^{\prime} \in X$ with $x \leq x^{\prime}$ and $y \leq f\left(x^{\prime}\right)$.

(4) For every upset $A \subseteq Y$, we have $f^{-1}(\downarrow A)=\downarrow f^{-1}(A)$.

(5) For every $y \in Y$, we have $f^{-1}(\downarrow \uparrow y)=\downarrow f^{-1}(\uparrow y)$.

Proof. (1) $\Rightarrow(2)$ : This is obvious.

$(2) \Rightarrow(3)$ : Suppose $x \in X, y \in Y$, and $f(x) \leq y$. Then $y \in \uparrow f(x)$. Since $Y$ is a Priestley space, there is $y^{\prime} \in \max \uparrow f(x)$ with $y \leq y^{\prime}$. By (2), $y^{\prime} \in f(\max \uparrow x)$. Therefore, there is $x^{\prime} \in X$ such that $x \leq x^{\prime}$ and $f\left(x^{\prime}\right)=y^{\prime} \geq y$. This shows (3).

$(3) \Rightarrow(1)$ : Let $x \in X$. We first show that $\max \uparrow f(x) \subseteq f(\max \uparrow x)$. Suppose $y \in \max \uparrow f(x)$. By (3), there is $x^{\prime} \in X$ with $x \leq x^{\prime}$ and $y \leq f\left(x^{\prime}\right)$. Since $y$ is a maximal point, $y=f\left(x^{\prime}\right)$. As $X$ is a Priestley space, there is $x^{\prime \prime} \in$ $\max \uparrow x$ with $x^{\prime} \leq x^{\prime \prime}$. Since $f$ is order preserving and $y$ is maximal, $f\left(x^{\prime \prime}\right)=$ $y$. Thus, $y \in f(\max \uparrow x)$. Next we show that $f(\max \uparrow x) \subseteq \max \uparrow f(x)$. First note that every map satisfying (3) maps maximal points to maximal points. For let $x \in X$ be maximal and suppose $x$ is mapped to a non-maximal $y \in Y$. Then there is $y^{\prime}$ in $Y$ with $y<y^{\prime}$. However, since $x$ is maximal, there is no $x^{\prime}$ with $x \leq x^{\prime}$ that is mapped to or above $y^{\prime}$. So Condition (3) is violated for $x$. Now suppose $y \in f(\max \uparrow x)$. Then there is $x^{\prime} \in \max \uparrow x$ such that $f\left(x^{\prime}\right)=y$. Since $f$ is order preserving, $f(x) \leq f\left(x^{\prime}\right)$. By the above, $x^{\prime}$ is mapped to a maximal point, so $f\left(x^{\prime}\right)=y \in \max \uparrow f(x)$. Thus, $f$ is a q-morphism.

$(3) \Rightarrow(4)$ : Let $A \subseteq Y$ be an upset. Since $f$ is order preserving, $\downarrow f^{-1}(A) \subseteq$ $f^{-1}(\downarrow A)$. We show $f^{-1}(\downarrow A) \subseteq \downarrow f^{-1}(A)$. Suppose $x \in f^{-1}(\downarrow A)$. Then there is $y \in A$ with $f(x) \leq y$. By $(3)$, there is $x^{\prime} \geq x$ with $y \leq f\left(x^{\prime}\right)$. Since $A$ is an upset, $f\left(x^{\prime}\right) \in A$, yielding $x \in \downarrow f^{-1}(A)$.

(4) $\Rightarrow(5)$ : This is obvious since $\uparrow y$ is an upset. 
$(5) \Rightarrow(3)$ : Let $x \in X, y \in Y$, and $f(x) \leq y$. Then $f(x) \in \downarrow \uparrow y$ so $x \in f^{-1}(\downarrow \uparrow y)$. By (5), $x \in \downarrow f^{-1}(\uparrow y)$. This implies that there is $x^{\prime} \geq x$ with $y \leq f\left(x^{\prime}\right)$, which proves $(3)$.

REMARK 7.4. Conditions (1) and (2) are not locally equivalent; that is, it is not true that for a given $x \in X$ we have $\max \uparrow f(x)=f(\max \uparrow x)$ iff $\max \uparrow f(x) \subseteq f(\max \uparrow x)$.

We are ready to see that the converse of Lemma 7.2 does not hold. For this we must find Heyting algebras $A, B$ such that $A$ is finite and s.i., $B$ is wellconnected, $B \not \forall \delta(A)$, and yet $A$ is not isomorphic to a pseudocomplemented sublattice of $B$. By Proposition 7.1, $B \not \models \delta(A)$ iff there is a s.i. homomorphic image $C$ of $B$ such that $A$ is isomorphic to a pseudocomplemented sublattice of $C$. Thus, we want $A$ to be isomorphic to a pseudocomplemented sublattice of a s.i. homomorphic image $C$ of $B$, and yet not to be isomorphic to a pseudocomplemented sublattice of $B$ itself. We do this by passing to the dual picture.

Dually finite Heyting algebras correspond to finite posets (partially ordered sets). If $A$ is a finite Heyting algebra and $X$ is its dual finite poset, then homomorphic images of $A$ correspond to upsets of $X$, subalgebras of $A$ to p-morphic images of $X$, pseudocomplemented sublattices of $A$ to q-morphic images of $X$, and bounded sublattices of $A$ to order preserving images of $X$ (see $[12,24,25])$. In addition, $A$ is s.i. iff $A$ is well-connected, which happens iff $X$ is rooted (that is, $X=\uparrow x$ for some $x \in X$ ) [3,13]. Therefore, it is sufficient to find two finite rooted posets $X, Y$ such that $X$ is a q-morphic image of an upset $U$ of $Y$, but $X$ is not a q-morphic image of $Y$.

EXAmple 7.5. Let $X$ and $Y$ be the finite rooted posets drawn below.

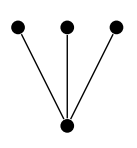

$X$

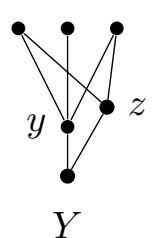

Y

Clearly $X$ is isomorphic to the upset $\uparrow y$ of $Y$. Therefore, $X$ is a q-morphic image of an upset of $Y$. On the other hand, we show that $X$ is not a qmorphic image of $Y$. First observe that an onto q-morphism $f: Y \rightarrow X$ would map the maximal points of $Y$ onto the maximal points of $X$. Now, $z \in Y$ sees two maximal points of $Y$, so since $f$ is order preserving, $f(z)$ would need to be the root of $X$. But as $X$ has three maximal points, the 
maximal points above $z$ are not mapped onto the maximal points above $f(z)$. Thus, $f$ cannot be a q-morphism.

We next define cofinal stable si-logics by directly generalizing the definition of stable si-logics of [5].

Definition 7.6. We call a si-logic $L$ cofinal stable provided for all s.i. Heyting algebras $A, B$, if $A$ is isomorphic to a pseudocomplemented sublattice of $B$ and $B \models L$, then $A \models L$.

Proposition 7.7. If $L$ is cofinal stable, then $L$ is axiomatizable by cofinal stable formulas.

Proof. We prove that $L=\mathbf{I P C}+\{\delta(A) \mid A$ finite and s.i., $A \not \models L\}$. Suppose that $B$ is a s.i. Heyting algebra with $B=L$. Let $A$ be a finite s.i. Heyting algebra with $A \not \models L$. If $B \not \models \delta(A)$, then by Proposition 7.1, there is a s.i. homomorphic image $C$ of $B$ such that $A$ is isomorphic to a pseudocomplemented sublattice of $C$. Since $B \models L$ and $C$ is a homomorphic image of $B$, we see that $C \models L$. As $L$ is cofinal stable, from $C \models L$ it follows that $A \models L$. The obtained contradiction proves that $B=\delta(A)$. Conversely, suppose that $B$ is a s.i. Heyting algebra with $B \not \models L$. Then there is $\varphi \in L$ with $B \not \models \varphi$. As in the proof of [5, Lem. 3.6], but using that the variety of pseudocomplemented distributive lattices is locally finite, we can construct a finite s.i. Heyting algebra $A$ such that $A$ is a pseudocomplemented sublattice of $B$ and $A \not \models \varphi$. Therefore, $A \not \models L$. Using Proposition 7.1 again yields $B \not \models \delta(A)$.

REMARK 7.8. The proof above also shows that all cofinal stable logics have the FMP.

Unlike the case of stable logics, the converse of Proposition 7.7 does not hold in general, as the following example shows.

ExAmple 7.9. Let $X, Y, Z$ be the finite rooted posets drawn below.
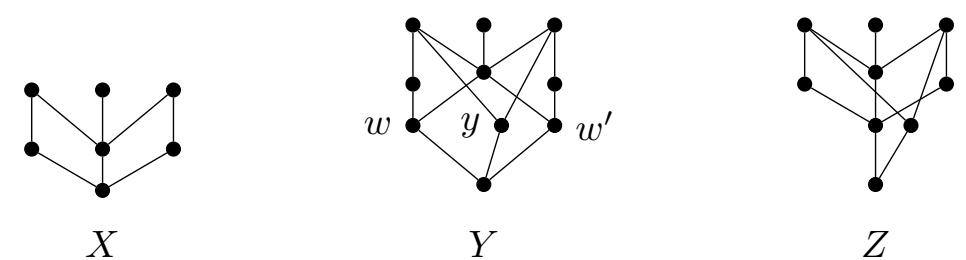

Let $A, B, C$ be the dual s.i. Heyting algebras of $X, Y, Z$, respectively, and let $L=\mathbf{I P C}+\delta(A)$. Then $L$ is axiomatized by a cofinal stable formula. On the other hand, we show that $L$ is not cofinal stable. First observe that $X$ 
is not a q-morphic image of $Y$. Indeed, if there were an onto q-morphism $f: Y \rightarrow X$, then $f$ would map the maximal points of $Y$ onto the maximal points of $X$. But $y \in Y$ sees exactly two maximal points, while $X$ has no point with this property. This violates the q-morphism condition at $y$, a contradiction. Since each proper rooted upset of $Y$ has smaller cardinality than $X$, it follows that there is no rooted upset $U$ of $Y$ such that $X$ is a q-morphic image of $U$. From this we conclude by Proposition 7.1 that $B=\delta(A)$, so $B=L$. On the other hand, $Z$ is a q-morphic image of $Y$, witnessed by the map that identifies $w$ and $w^{\prime}$ in $Y$. Therefore, $C$ is isomorphic to a pseudocomplemented sublattice of $B$. It is also obvious that $X$ is isomorphic to an upset of $Z$, so by Proposition 7.1, $C \not \models \delta(A)$. Thus, $C \not \models L$. Since $B \models L, C$ is isomorphic to a pseudocomplemented sublattice of $B$, and $C \not \models L$, we conclude that $L$ is not a cofinal stable logic.

REMARK 7.10. Although as pointed out in Remark 7.8 cofinal stable logics have the FMP, we do not know whether all logics axiomatized by cofinal stable formulas have the FMP. Neither do we know a convenient characterization of this class of logics.

As follows from Example 7.9, Theorem 5.3 does not fully generalize to the setting of cofinal stable logics as the analogue of Condition (2) of the theorem is not equivalent to Condition (1). Nevertheless, we will prove that all the conditions of the theorem but Condition (2) are still equivalent in the cofinal stable setting. In fact, some of the proofs will have a straightforward generalization, while others will require additional work.

TheOREM 7.11. Let $L$ be a si-logic and let $\mathcal{V}$ be the corresponding variety of Heyting algebras. The following are equivalent.

$\left(1^{\prime}\right) L$ is a cofinal stable logic.

$\left(2^{\prime}\right) \mathcal{V}_{w c}$ is cofinal stable.

$\left(3^{\prime}\right) \mathcal{V}$ is generated by a cofinal stable universal class $\mathcal{U}$.

$\left(4^{\prime}\right) \mathcal{V}$ is generated by a cofinal stable class $\mathcal{K}$.

$\left(5^{\prime}\right) L$ is axiomatizable by cofinal stable rules.

Proof. The proof of $\left(2^{\prime}\right) \Rightarrow\left(3^{\prime}\right)$ is the same as the proof of $(4) \Rightarrow(5)$ in Theorem 5.3, and the proof of $\left(3^{\prime}\right) \Rightarrow\left(4^{\prime}\right)$ is obvious.

$\left(4^{\prime}\right) \Rightarrow\left(2^{\prime}\right)$ : Let $\mathcal{V}$ be generated by a cofinal stable class $\mathcal{K}$. Suppose $B, C$ are Heyting algebras such that $C \in \mathcal{V}_{w c}$ and $B$ is isomorphic to a pseudocomplemented sublattice of $C$. Then $B$ is also well-connected, so it remains to prove that $B \in \mathcal{V}$. If $B \notin \mathcal{V}$, then there is $\varphi \in L$ with $B \not \forall \varphi$. 
We can find a finite pseudocomplemented sublattice $B^{\prime}$ of $B$ with $B^{\prime} \not \models \varphi$. Clearly $B^{\prime} \not \neq L$. Since $B$ is well-connected, so is $B^{\prime}$, and as $B^{\prime}$ is finite, it is s.i. Next we require an analogue of Claim 5.5. But the proof in the stable case makes use of [5, Lem. 6.2], whose analogue, as we saw, does not hold in the cofinal stable case. Nevertheless, we can derive the desired result using the following claim instead.

Claim 7.12. Suppose $A, B$ are finite Heyting algebras such that $A$ is isomorphic to a pseudocomplemented sublattice of a homomorphic image of $B$. Then there is a finite Heyting algebra $C$ such that $C$ is isomorphic to a pseudocomplemented sublattice of $B$ and $A$ is a homomorphic image of $C$.

Proof of Claim. Suppose $A$ is isomorphic to a pseudocomplemented sublattice of a homomorphic image of $B$. Since $B$ is finite, homomorphic images of $B$ are up to isomorphism quotients of $B$ by principal filters. Clearly $B / \uparrow b \cong[0, b]$, and the homomorphism $h: B \rightarrow[0, b]$ is given by $h(x)=b \wedge x$. Therefore, we may assume that $A$ is isomorphic to a pseudocomplemented sublattice of $[0, b]$, and we identify $A$ with its image in $[0, b]$. Let $C=h^{-1}(A)$. It is obvious that $C$ is a pseudocomplemented sublattice of $B$. We claim that the restriction of $h$ to $C$ is a Heyting homomorphism onto $A$. For this it is sufficient to check that $h$ preserves Heyting implication. Let $x, y \in C$. Since $C$ is finite and $h$ commutes with joins,

$$
\begin{aligned}
h\left(x \rightarrow_{C} y\right) & =\bigvee_{A}\{h(c) \mid c \in C \text { and } x \wedge c \leq y\} \\
& =\bigvee_{A}\{c \wedge b \mid c \in C \text { and } x \wedge c \leq y\}
\end{aligned}
$$

and

$$
h(x) \rightarrow_{A} h(y)=\bigvee_{A}\{z \in A \mid(x \wedge b) \wedge z \leq y\} .
$$

It is easy to see that $\{c \wedge b \mid c \in C$ and $x \wedge c \leq y\} \subseteq\{z \in A \mid(x \wedge b) \wedge z \leq y\}$. For the reverse inclusion, let $z \in A$ with $(x \wedge b) \wedge z \leq y$. Since $z \in A \subseteq[0, b]$, we see that $z=z \wedge b=h(z)$, so $z \in C$ and $x \wedge z=x \wedge(b \wedge z) \leq y$. This shows that $\{z \in A \mid(x \wedge b) \wedge z \leq y\} \subseteq\{c \wedge b \mid c \in C$ and $x \wedge c \leq y\}$. Therefore, $h\left(x \rightarrow_{C} y\right)=h(x) \rightarrow_{A} h(y)$. Thus, the restriction of $h$ to $C$ is a Heyting homomorphism and it is clearly onto.

Claim 7.13. If $\mathcal{V}$ is generated by a cofinal stable class $\mathcal{K}$, then for every finite s.i.Heyting algebra $A$, from $A \not \models L$ it follows that $\delta(A) \in L$.

Proof of Claim. Suppose $A$ is a finite s.i. Heyting algebra and $A \not \models L$. Since $\mathcal{V}$ is generated by a cofinal stable class $\mathcal{K}$, a similar argument to 
Claim 5.4 yields that each non-theorem of $L$ is refuted on a finite member of $\mathcal{K}$. In particular, $L$ has the FMP. Therefore, if $\delta(A) \notin L$, then there is a finite $B \in \mathcal{K}$ with $B \not \models \delta(A)$. By Proposition 7.1, there is a s.i. homomorphic image $B^{\prime}$ of $B$ such that $A$ is isomorphic to a pseudocomplemented sublattice of $B^{\prime}$. By Claim 7.12, there is a Heyting algebra $A^{\prime}$ such that $A^{\prime}$ is isomorphic to a pseudocomplemented sublattice of $B$ and $A$ is a homomorphic image of $A^{\prime}$. Since $B \in \mathcal{K}$ and $\mathcal{K}$ is cofinal stable, $A^{\prime} \in \mathcal{K}$, so $A^{\prime} \models L$. As $A$ is a homomorphic image of $A^{\prime}$, we see that $A=L$. The obtained contradiction proves that $\delta(A) \in L$.

We are ready to complete the proof of $\left(4^{\prime}\right) \Rightarrow\left(2^{\prime}\right)$. Since $B^{\prime}$ is a finite s.i. Heyting algebra such that $B^{\prime} \not \models L$, by Claim $7.13, \delta\left(B^{\prime}\right) \in L$. As $C$ is well-connected and $B^{\prime}$ is isomorphic to a pseudocomplemented sublattice of $C$, Lemma 7.2 yields $C \not \models \delta\left(B^{\prime}\right)$. Since $\delta\left(B^{\prime}\right) \in L$, this contradicts $C \models L$. The obtained contradiction proves that $B \in \mathcal{V}$.

$\left(3^{\prime}\right) \Rightarrow\left(5^{\prime}\right)$ : Suppose $\mathcal{V}$ is generated by a cofinal stable universal class $\mathcal{U}$. Let $\mathcal{S}$ be the intuitionistic multi-conclusion consequence relation corresponding to $\mathcal{U}$. By Proposition $6.5, \mathcal{S}=\mathcal{S}_{\text {IPC }}+\{\sigma(A) \mid A$ finite, $A \notin \mathcal{U}\}$. Therefore, since $\mathcal{V}$ is generated by $\mathcal{U}$, we see that $L=\Lambda(\mathcal{S})$. Thus, $L$ is axiomatizable by cofinal stable rules.

$\left(5^{\prime}\right) \Rightarrow\left(3^{\prime}\right)$ : Suppose $L$ is axiomatizable by cofinal stable rules. Then there is a family $\left\{A_{i} \mid i \in I\right\}$ of finite Heyting algebras such that $L=\Lambda(\mathcal{S})$, where $\mathcal{S}=\mathcal{S}_{\text {IPC }}+\left\{\sigma\left(A_{i}\right) \mid i \in I\right\}$. Therefore, $\mathcal{V}$ is generated by $\mathcal{U}(\mathcal{S})$, which by Proposition 6.5 is a cofinal stable universal class.

The proof of $\left(2^{\prime}\right) \Rightarrow\left(1^{\prime}\right)$ is easy.

$\left(1^{\prime}\right) \Rightarrow\left(4^{\prime}\right)$ : Let $\mathcal{V}_{f s i}$ be the class of finite s.i. members of $\mathcal{V}$. We show that $\mathcal{V}_{f s i}$ is a cofinal stable class generating $\mathcal{V}$. Since $L$ is cofinal stable, as we pointed out in Remark 7.8, $L$ has the FMP. Therefore, $\mathcal{V}$ is generated by $\mathcal{V}_{f s i}$. To see that $\mathcal{V}_{f s i}$ is cofinal stable, let $A, B$ be Heyting algebras such that $A$ is isomorphic to a pseudocomplemented sublattice of $B$ and $B \in \mathcal{V}_{f s i}$. Since $B$ is finite, $B$ is well-connected, so $A$ is well-connected, and as $A$ is finite, we conclude that $A$ is s.i. Therefore, by $\left(1^{\prime}\right), A \in \mathcal{V}_{f s i}$, showing that $\mathcal{V}_{f s i}$ is cofinal stable.

REMARK 7.14. The analogue of Remark 5.7 does not hold in the cofinal stable case. To see this, let $X, Y, Z$ be the finite rooted posets of Example 7.9 and let $A, B, C$ be their dual s.i. Heyting algebras. It is easy to see that $X$ is not a q-morphic image of $Z$. Therefore, $C \models \sigma(A)$, so $C \models \Lambda\left(\mathcal{S}_{\text {IPC }}+\sigma(A)\right)$. On the other hand, we saw in Example 7.9 that $C \not \models \delta(A)$. Thus, IPC $+\delta(A) \neq \Lambda\left(\mathcal{S}_{\text {IPC }}+\sigma(A)\right)$. The same example shows that Remark 5.7 cannot be generalized to $(\wedge, \vee)$-canonical formulas and 
rules. In fact, the same reasoning as above shows that IPC $+\gamma(A, D) \neq$ $\Lambda\left(\mathcal{S}_{\text {IPC }}+\rho(A, D)\right)$, where $D=\{(a, 0) \mid a \in A\} \subseteq A^{2}$.

\section{Cardinality}

It is clear that each stable multi-conclusion consequence relation is a cofinal stable multi-conclusion consequence relation, and that each stable logic is a cofinal stable logic, but the converse is not true in general. It was shown in [5, Thm. 6.13] that there are continuum many stable logics. We show that there are continuum many cofinal stable logics that are not stable logics. It follows that there are continuum many cofinal stable multi-conclusion consequence relations that are not stable multi-conclusion consequence relations.

Consider the sequence of finite posets drawn below. It is well known that no member of this sequence is a p-morphic image of an upset of some other member. We will show that the same result holds if we replace p-morphisms with q-morphisms.

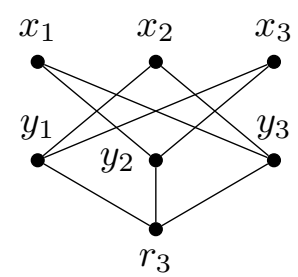

$X_{3}$

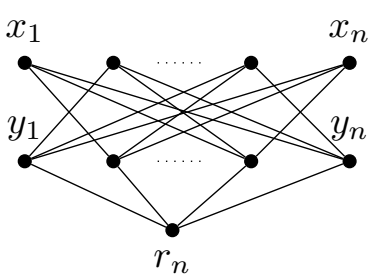

$X_{n}$

In each $X_{n}$ we have $x_{i} \leq y_{j}$ iff $i \neq j$ and $r_{n}$ is the root.

Lemma 8.1. For $n, m \geq 3$, if $n \neq m$, then $X_{n}$ is not a q-morphic image of any upset of $X_{m}$.

Proof. Let $m, n \geq 3$ with $n \neq m$. Clearly if $m<n$, then $X_{n}$ cannot be a q-morphic image of an upset of $X_{m}$. Suppose $n<m$. Let $r_{n}$ be the root of $X_{n}$ and $r_{m}$ be the root of $X_{m}$. Also, let $x_{i}, y_{i}$ be the elements of $X_{n}$ and $x_{i}^{\prime}, y_{i}^{\prime}$ be the elements of $X_{m}$. Suppose there is an onto q-morphism $f$ from an upset $U$ of $X_{m}$ onto $X_{n}$. First we show that $\max \left(X_{m}\right) \subseteq U$. Since $f$ is an onto q-morphism, the maximal elements of $U$ have to be mapped onto the maximal elements of $X_{n}$, so $f(\max U)=\max \left(X_{n}\right)$. As $n \geq 3$, this implies that $U$ contains $y_{i}^{\prime}, y_{j}^{\prime}$ for $i \neq j$. But $\max \left(X_{m}\right) \subseteq \uparrow\left\{y_{i}^{\prime}, y_{j}^{\prime}\right\} \subseteq U$. Next we observe that no $y_{i}^{\prime} \in U$ is mapped to some $x_{j}$. For suppose there is $y_{i}^{\prime} \in U$ with $f\left(y_{i}^{\prime}\right)=x_{j}$. Since $y_{i}^{\prime}$ sees all but one maximal element of $X_{m}$, 
we see that $f$ maps all but one maximal element to $x_{j}$. As $X_{n}$ has at least 3 maximal elements, this contradicts the fact that $f\left(\max X_{m}\right)=\max \left(X_{n}\right)$. Therefore, each $y_{i}^{\prime} \in U$ is mapped to some $y_{j}$ or to $r_{n}$. Now suppose $f$ maps two maximal elements $x_{i}^{\prime}$ and $x_{j}^{\prime}$ to the same maximal element $x_{k}$. If $y_{i}^{\prime} \in U$, then $\max \uparrow f\left(y_{i}^{\prime}\right)=f\left(\max \uparrow y_{i}^{\prime}\right)=\max \left(X_{n}\right)$. This means that $f$ maps $y_{i}^{\prime}$ to the root $r_{n}$. A similar argument gives that if $y_{j}^{\prime} \in U$, then $f\left(y_{j}^{\prime}\right)=r_{n}$. Since $m>n$, by the pigeonhole principle, there is at least one maximal element of $X_{n}$ that has two $f$-preimages. Therefore, there are at most $n-1$ maximal elements of $X_{n}$ that have a unique $f$-preimage. This in turn means that there are at most $n-1$ candidates $y_{i} \in U$ that $f$ could map to some element of depth 2 of $X_{n}$. But then $f$ cannot be onto. The obtained contradiction proves that there is no q-morphism from an upset of $X_{m}$ onto $X_{n}$.

On the other hand, we have the following lemma.

Lemma 8.2. If $m \geq 2 n$, then there is an onto order preserving map $f$ : $X_{m} \rightarrow X_{n}$.

Proof. Using the same notation as in the previous lemma, define $f: X_{m} \rightarrow$ $X_{n}$ as follows. For $i \leq n$, send $x_{i}^{\prime}$ to $x_{i}, x_{n+i}^{\prime}$ to $y_{i}$, and send the rest of the points in $X_{m}$ to the root $r_{n}$ of $X_{n}$. It is straightforward to see that such $f$ is an onto order preserving map.

We are ready to prove the main result of this section. To simplify notation, for $n \geq 3$, we denote the cofinal stable formula of the dual Heyting algebra of $X_{n}$ by $\delta\left(X_{n}\right)$.

Proposition 8.3. There are continuum many cofinal stable si-logics that are not stable.

Proof. For $n \geq 3$, suppose $\mathcal{K}_{n}$ is the class of q-morphic images of $X_{n}$. Let $I=\{n \in \mathbb{N} \mid n \geq 3\}$, and for $J \subseteq I$, let $L(J)$ be the logic of $\bigcup_{n \in J} \mathcal{K}_{n}$. Since $\bigcup_{n \in J} \mathcal{K}_{n}$ is closed under q-morphic images, the corresponding class of finite Heyting algebras is cofinal stable. Thus, $L(J)$ is a cofinal stable logic.

Claim 8.4. For $S, T \subseteq I$, if $S \neq T$, then $L(S) \neq L(T)$.

ProOF OF ClAIM. We may assume without loss of generality that there is $n \in S \backslash T$. Then $X_{n} \models L(S)$, which implies that $\delta\left(X_{n}\right) \notin L(S)$ since $X_{n} \not \models$ $\delta\left(X_{n}\right)$. We prove that $\delta\left(X_{n}\right) \in L(T)$. If $\delta\left(X_{n}\right) \notin L(T)$, then there is $m \in T$ and $Y \in \mathcal{K}_{m}$ with $Y \not \models \delta\left(X_{n}\right)$. By the dual statement of Proposition 7.1, there is a rooted upset $Z$ of $Y$ such that $X_{n}$ is a q-morphic image of $Z$. Now $Y \in \mathcal{K}_{m}$ means that $Y$ is a q-morphic image of $X_{m}$. Let $f: X_{m} \rightarrow Y$ be an onto q-morphism, and let $U=f^{-1}(Z)$. Then $U$ is an upset of $X_{m}$ and the 
restriction of $f$ to $U$ is a q-morphism onto $Z$. Therefore, $Z$ is a q-morphic image of an upset of $X_{m}$, and hence $X_{n}$ is a q-morphic image of an upset of $X_{m}$. Since $n \notin T$, we have $m \neq n$. This contradicts Lemma 8.1. Thus, $\delta\left(X_{n}\right) \in L(T)$, and so $L(S) \neq L(T)$.

Let $\Delta=\{J \subseteq I \mid J$ and $I \backslash J$ are infinite $\}$. By Claim 8.4, $\{L(J) \mid J \in \Delta\}$ provides continuum many cofinal stable logics. It remains to be shown that $L(J)$ is not stable for each $J \in \Delta$. Let $J \in \Delta$. Then there is $n \in I \backslash J$. By the proof of Claim 8.4, $\delta\left(X_{n}\right) \in L(J)$. Therefore, since $X_{n} \not \models \delta\left(X_{n}\right)$, we see that $X_{n} \not \neq L(J)$. Now there is $m \in J$ with $m \geq 2 n$. As $m \in J$, we have $X_{m} \models L(J)$. By Lemma 8.2, $X_{n}$ is a stable image of $X_{m}$. Because $X_{n} \not \models L(J)$, we conclude that $L(J)$ is not stable.

REMARK 8.5. Let $Y_{n}$ be obtained from $X_{n}$ by removing the root. Then the same proof as above, but using the cofinal stable rules $\sigma\left(Y_{n}\right)$ instead of the cofinal stable formulas $\delta\left(X_{n}\right)$ yields a continuum of cofinal stable multi-conclusion consequence relations of depth 2 . This shows that cofinal stable multi-conclusion consequence relations have much more complicated structure than cofinal stable logics as there are only countably many cofinal stable logics of depth 2 (see below).

\section{Examples}

In this final section we give examples comparing cofinal stable, stable, subframe, and cofinal subframe si-logics. In the previous section we saw that there are continuum many cofinal stable logics that are not stable. In this section we show that each logic of depth 2 is a cofinal stable logic, while none of these but one is a stable logic. We prove that Maksimova's logics $\mathbf{N D}_{k}$ provide a family of cofinal stable logics that are neither stable nor cofinal subframe for $k \geq 2$. Examples of stable logics that are neither splitting nor subframe logics, as well as examples of splitting and subframe logics that are not stable logics were given in [5, Thm. 7.4]. We give an example of a stable logic that is not a cofinal subframe logic.

For a finite poset $X$, let $\operatorname{depth}(X)$ denote the depth of $X$. We recall that $\operatorname{depth}(X)=n$ if $X$ contains a chain of length $n$, but no chain of bigger length. Let $\mathbf{B D}_{n}$ be the si-logic of all finite ordered sets of depth $\leq n$ and let $\mathbf{L C}_{n}$ be the si-logic of finite chains of depth $\leq n$. Then $\mathbf{C P C}=\mathbf{B D}_{1}=\mathbf{L C}_{1}$, and a si-logic $L$ is of depth $n$ iff $\mathbf{B D}_{n} \subseteq L \subseteq \mathbf{L} \mathbf{C}_{n}$.

It was shown in [5, Thm. 7.4] that each $\mathbf{B D}_{n}$, for $n \geq 2$, is not stable. 
Proposition 9.1. Suppose $L$ is a si-logic containing $\mathbf{B D}_{2}$. Then $L$ is cofinal stable, and it is stable iff $\mathbf{L C}_{2} \subseteq L$.

Proof. It is well known (see, e.g., [17]) that $\mathbf{B D}_{2}$ is the logic of all finite forks $\mathfrak{F}_{n}$ drawn below, that $\mathbf{L C}_{2}$ is the logic of the two element chain $\mathfrak{F}_{1}$, and that each $L$ in the interval $\left(\mathbf{B D}_{2}, \mathbf{L} \mathbf{C}_{2}\right]$ is the logic of the $n$-fork $\mathfrak{F}_{n}$ for some $n \geq 1$.

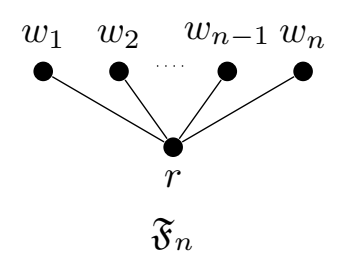

It follows from the proof of [5, Thm. 7.4] that no si-logic $L$ that contains $\mathbf{B D}_{2}$ and is properly contained in $\mathbf{L} \mathbf{C}_{2}$ is stable. The only other si-logics containing $\mathbf{B D}_{2}$ are $\mathbf{L} \mathbf{C}_{2}, \mathbf{C P C}$, and the inconsistent logic. It is easy to see that each of these is stable. We prove that each $L$ containing $\mathbf{B D}_{2}$ is cofinal stable. Since q-morphisms map maximal elements to maximal elements, it is immediate to see that every q-morphic image of the $n$-fork is either the one-point poset or the $m$-fork for $m \leq n$. Therefore, for each $L \supseteq \mathbf{B D}_{2}$, the class of finite rooted $L$-frames is closed under q-morphic images. This means that the class of finite s.i. $L$-algebras is cofinal stable, and it generates the variety corresponding to $L$. Thus, by Theorem 7.11, $L \supseteq \mathbf{B D}_{2}$ is cofinal stable.

We next show that $\mathbf{B D}_{2}$ can be axiomatized by the cofinal stable formula of the dual Heyting algebra of the three-element chain $\mathfrak{C}_{3}=\left\{w_{1}, w_{2}, w_{3}\right\}$, where $w_{1}<w_{2}<w_{3}$. For convenience, we write $\delta\left(\mathfrak{C}_{3}\right)$ for the cofinal stable formula of the Heyting algebra dual to $\mathfrak{C}_{3}$. (Clearly this Heyting algebra is the four-element chain.)

Lemma 9.2. Suppose $X$ is a finite rooted poset. Then $\operatorname{depth}(X) \leq 2$ iff $\mathfrak{C}_{3}$ is not a q-morphic image of $X$.

Proof. We prove that $\operatorname{depth}(X) \geq 3$ iff $\mathfrak{C}_{3}$ is a q-morphic image of $X$. First suppose that $\operatorname{depth}(X) \geq 3$. Then $X$ contains a chain $x_{1}<x_{2}<x_{3}$. Define $f: X \rightarrow \mathfrak{C}_{3}$ by setting

$$
f(y)= \begin{cases}w_{1} & \text { if } y \in \downarrow x_{1} \\ w_{2} & \text { if } y \in \downarrow x_{2} \backslash \downarrow x_{1}, \\ w_{3} & \text { otherwise. }\end{cases}
$$


It is easy to see that $f$ is an onto q-morphism (in fact, it is an onto pmorphism). Conversely, suppose $f: X \rightarrow \mathfrak{C}_{3}$ is an onto q-morphism. We show that $X$ contains a chain of three elements. Since $f$ is onto, the root $r$ of $X$ is mapped by $f$ to $w_{1}$. Using again that $f$ is onto, we find $x>r$ with $f(x)=w_{2}$. Since $f(x)=w_{2}<w_{3}$ and $w_{3} \in \max \left(\mathfrak{C}_{3}\right)$, there is $y>x$ such that $f(y)=w_{3}$. Thus, $r<x<y$ is a three element chain in $X$.

Proposition 9.3. $\mathbf{B D}_{2}=\mathbf{I P C}+\delta\left(\mathfrak{C}_{3}\right)$.

Proof. Suppose $\mathfrak{F}_{n}$ is the $n$-fork. By Lemma $9.2, \mathfrak{C}_{3}$ is not a q-morphic image of a rooted upset of $\mathfrak{F}_{n}$. By the dual statement of Proposition 7.1, $\mathfrak{F}_{n} \models \delta\left(\mathfrak{C}_{3}\right)$. Since $\mathbf{B D}_{2}$ is the logic of all $n$-forks, we conclude that IPC + $\delta\left(\mathfrak{C}_{3}\right) \subseteq \mathbf{B D}_{2}$. Conversely, suppose $X$ is an Esakia space such that $X \not \models$ $\mathbf{B D}_{2}$. Since $\mathbf{B D}_{2}=\mathbf{I P C}+\mathbf{b d}_{\mathbf{2}}$, where $\mathbf{b d}_{\mathbf{2}}=q \vee(q \rightarrow(p \vee \neg p))$, we see that $X \not \neq \mathbf{b d}_{\mathbf{2}}$. By dualizing the end of the proof of Proposition 7.7, we can construct a finite rooted poset $Y$ such that $Y$ is a continuous q-morphic image of $X$ and $Y \not \models \mathbf{b d}_{\mathbf{2}}$. It follows that $\boldsymbol{d e p t h}(Y) \geq 3$. By Lemma 9.2, $\mathfrak{C}_{3}$ is a q-morphic image of $Y$. Therefore, $\mathfrak{C}_{3}$ is a continuous q-morphic image of $X$. Thus, by the dual statement of Proposition 7.1, $X \not \models \delta\left(\mathfrak{C}_{3}\right)$. As every si-logic $L$ is complete with respect to the Esakia spaces validating $L$, we conclude that $\mathbf{B D}_{2} \subseteq \mathbf{I P C}+\delta\left(\mathfrak{C}_{3}\right)$.

For $n \geq 3$, the logics $\mathbf{B D}_{n}$ are neither stable nor cofinal stable. The following picture shows an onto q-morphism from a rooted poset of depth 3 to a rooted poset of depth 4 . We infer that the class of finite rooted posets of depth 3 is not closed under q-morphic images, which entails that $\mathbf{B D}_{3}$ is not cofinal stable. Clearly similar examples can be constructed to show that $\mathbf{B D}_{n}$ is not cofinal stable for all $n \geq 3$.

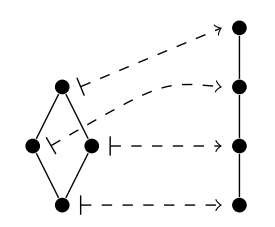

Next we consider the logics $\mathbf{N D}_{k}$ introduced by Maksiomova [22] (see also [16, Sec. 2.4]). For $k \in \mathbb{N}$, let

$$
\mathbf{n d}_{k}=\left(\neg p \rightarrow \bigvee_{1 \leq i \leq k} \neg q_{i}\right) \rightarrow \bigvee_{1 \leq i \leq k}\left(\neg p \rightarrow \neg q_{i}\right),
$$

and let $\mathbf{N D}_{k}=\mathbf{I P C}+\mathbf{n d} \mathbf{d}_{k}$. As was pointed out in [22], each $\mathbf{N D} \mathbf{D}_{k}$ has the FMP. On finite frames, $\mathbf{n d}_{k}$ characterizes the property of having divergence 
$k$, where a finite poset $X$ is of divergence $k$ if for all $x \in X$ and $W \subseteq \max (X)$ satisfying $|W| \leq k$ and $W \subseteq \uparrow x$, there is $y \geq x$ with $\max (\uparrow y)=W$. Therefore, $\mathbf{N D}_{k}$ is the logic of the finite frames of divergence $k$.

We show that each $\mathbf{N D}_{k}$ is a cofinal stable logic, but that for $k \geq 2$, $\mathbf{N D}_{k}$ is neither stable nor cofinal subframe logic (and hence not a subframe logic).

Lemma 9.4. Each $\mathbf{N D}_{k}$ is a cofinal stable logic.

Proof. Since $\mathbf{N D}_{k}$ has the FMP, it is sufficient to check that the finite frames of $\mathbf{N D}_{k}$ are closed under q-morphic images. Let $X$ be a finite poset of divergence $k$ and let $X^{\prime}$ be a q-morphic image of $X$ under the q-morphism $f$ : $X \rightarrow X^{\prime}$. We show that $X^{\prime}$ is of divergence $k$. Let $x^{\prime} \in X^{\prime}, W^{\prime} \subseteq \max \left(X^{\prime}\right)$, $\left|W^{\prime}\right| \leq k$, and $W^{\prime} \subseteq \uparrow x^{\prime}$. Since $f$ is onto, there is $x \in X$ with $f(x)=x^{\prime}$. As $f$ is a q-morphism, for each $w^{\prime} \in W^{\prime}$ we can pick one $w \in \max (X)$ with $x \leq w$ and $f(w)=w^{\prime}$. We denote the set of these elements by $W$. Then $|W|=\left|W^{\prime}\right| \leq k, W \subseteq \max (X)$, and $W \subseteq \uparrow x$. Since $X$ is of divergence $k$, there is $y \geq x$ with $\max (\uparrow y)=W$. Because $f$ is order-preserving, $x^{\prime} \leq^{\prime}$ $f(y)$, and by the q-morphism condition, $\max (\uparrow f(y))=W^{\prime}$. Thus, $X^{\prime}$ is of divergence $k$.

LEMMA 9.5. For $k \geq 2$, the logic $\mathbf{N D}_{k}$ is neither stable nor cofinal subframe logic. Consequently, $\mathbf{N D}_{k}$ is not a subframe logic.

Proof. Let $k \geq 2$, and consider the posets $X$ and $Y$ depicted below. It is easy to see that $X$ is of divergence $k$, while $Y$ is not of divergence 2. But $Y$ is both a stable image and a cofinal subframe of $Y$.

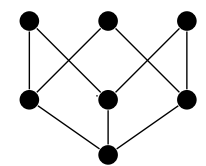

$X$

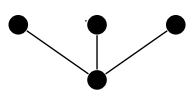

Y

Finally, we construct a tabular stable logic that is not a cofinal subframe logic. Let $\mathfrak{F}, \mathfrak{G}$ be the posets drawn below. We let $S t(\mathfrak{F})=\{\mathfrak{K} \mid$ $\mathfrak{K}$ is an order preserving image of $\mathfrak{F}\}$ and $\mathbf{L}_{S t(\mathfrak{F})}$ be the logic of $S t(\mathfrak{F})$. Since the class of Heyting algebras corresponding to the class $S t(\mathfrak{F})$ is stable, $\mathbf{L}_{S t(\mathfrak{F})}$ is a stable logic. 


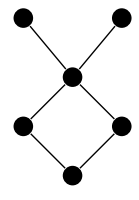

$\mathfrak{F}$

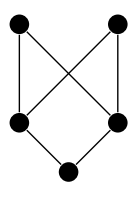

$\mathfrak{G}$

Lemma 9.6. The logic $\mathbf{L}_{S t(\mathfrak{F})}$ is not a cofinal subframe logic.

Proof. Suppose $\mathbf{L}_{S t(\mathfrak{F})}$ is a cofinal subframe logic. It is easy to see that $\mathfrak{G}$

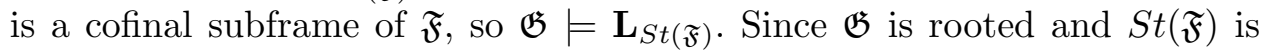
finite, the dual statement of Jónsson's Lemma yields that $\mathfrak{G}$ is isomorphic to an upset of a p-morphic image of a member of $S t(\mathfrak{F})$. But $S t(\mathfrak{F})$ is closed under order preserving images, hence it is closed under p-morphic images. Therefore, there is an onto order preserving map $f: \mathfrak{F} \rightarrow \mathfrak{K}$ such that $\mathfrak{G}$ is an upset of $\mathfrak{K}$. Since $\mathfrak{K}$ is an order preserving image of $\mathfrak{F}$, we see that $\mathfrak{K}$ has at most two maximal points. But $\mathfrak{G}$ is an upset of $\mathfrak{K}$, so $\mathfrak{K}$ has precisely two maximal points, and $f$ maps the maximal points of $\mathfrak{F}$ to the maximal points of $\mathfrak{K}$. Note that $\mathfrak{F}$ has only one element of depth 2 , which is a cover of the maximal elements of $\mathfrak{F}$. It follows that $\mathfrak{K}$ also has only one element of depth 2 . However, $\mathfrak{G}$ has two elements of depth 2 . Thus, $\mathfrak{G}$ cannot be an upset of $\mathfrak{K}$. The obtained contradiction proves that $\mathbf{L}_{S t(\mathfrak{F})}$ is not a cofinal subframe logic.

The following table summarizes the above comparison of stable, cofinal stable, subframe, and cofinal subframe logics.

\begin{tabular}{lcccc}
\hline & subframe & cof. subframe & stable & cof. stable \\
\hline $\mathbf{L C}_{n}$ & $\checkmark$ & $\checkmark$ & $\checkmark$ & $\checkmark$ \\
$\mathbf{B D}_{2}$ & $\checkmark$ & $\checkmark$ & - & $\checkmark$ \\
$\mathbf{B D}_{n}, n \geq 3$ & $\checkmark$ & $\checkmark$ & - & - \\
$\mathbf{N D}_{k}, k \geq 2$ & - & - & - & $\checkmark$ \\
$\mathbf{L}_{S t(\mathfrak{F})}$ & - & - & $\checkmark$ & $\checkmark$ \\
\hline
\end{tabular}

Acknowledgements. The authors would like to thank David Gabelaia and Mamuka Jibladze for inspiring and helpful discussions that took place during TOLO IV (International Workshop on Topological Methods in Logic) in Tbilisi, June 23-27, 2014.

Open Access. This article is distributed under the terms of the Creative Commons Attribution 4.0 International License (http://creativecommons. 
org/licenses/by/4.0/), which permits unrestricted use, distribution, and reproduction in any medium, provided you give appropriate credit to the original author(s) and the source, provide a link to the Creative Commons license, and indicate if changes were made.

\section{References}

[1] Balbes, R., and P. Dwinger, Distributive Lattices, University of Missouri Press, Columbia, MO, 1974.

[2] Bezhanishvili, G., Locally finite varieties, Algebra Universalis 46(4):531-548, 2001.

[3] Bezhanishvili, G., and N. Bezhanishvili, Profinite Heyting algebras, Order 25(3):211-227, 2008.

[4] Bezhanishvili, G., and N. Bezhanishvili, An algebraic approach to canonical formulas: Intuitionistic case, The Review of Symbolic Logic 2(3):517-549, 2009.

[5] Bezhanishvili, G., and N. Bezhanishvili, Locally finite reducts of Heyting algebras and canonical formulas. Notre Dame Journal of Formal Logic, to appear. Available at http://dspace.library.uu.nl/handle/1874/273468.

[6] Bezhanishvili, G., N. Bezhanishvili, and R. Iemhoff, Stable canonical rules, The Journal of Symbolic Logic 81(1):284-315, 2016.

[7] Burris, S., and H. P. Sankappanavar, A Course in Universal Algebra, Graduate Texts in Mathematics, Springer, 1981.

[8] Chagrov, A. V., and M. Zakharyaschev, Modal Logic, vol. 35 of Oxford Logic Guides. Oxford University Press, Oxford, 1997.

[9] Citkin, A., Characteristic inference rules, Logica Universalis 9(1):27-46, 2015.

[10] DE Jongh, D., Investigations on the Intuitionistic Propositional Calculus, PhD thesis, University of Wisconsin, 1968.

[11] Diego, A., Sur les Algèbres de Hilbert, Translated from the Spanish by Luisa Iturrioz. Collection de Logique Mathématique, Sér. A, Fasc. XXI, Gauthier-Villars, Paris, 1966.

[12] Esakia, L., Topological Kripke models, Soviet Mathematics Doklady 15:147-151, 1974.

[13] Esakia, L., On the theory of modal and superintuitionistic systems, in Logical Inference (Moscow, 1974), Nauka, Moscow, 1979, pp. 147-172 (Russian).

[14] Fine, K., An ascending sequence of S4 logics, Theoria 40(2):110-116, 1974.

[15] Fine, K., Logics containing K4. II, The Journal of Symbolic Logic 50(3):619-651, 1985.

[16] Goudsmit, J., Intuitionistic Rules. Admissible Rules of Intermediate Logics, $\mathrm{PhD}$ thesis, Utrecht University, 2015.

[17] Hosoi, T., and H. Ono, The intermediate logics on the second slice, Journal of the Faculty of Science, University of Tokyo, Tokyo, Section I A. Mathematics 17:457-461, 1970.

[18] Iemhoff, R., Consequence relations and admissible rules, The Journal of Philosophical Logic, 2016. doi:10.1007/s10992-015-9380-8.

[19] JANKOV, V., On the relation between deducibility in intuitionistic propositional calculus and finite implicative structures, Doklady Akademii Nauk SSSR 151:1293-1294, 1963 (Russian). 
[20] JANkov, V., The construction of a sequence of strongly independent superintuitionistic propositional calculi, Soviet Mathematics Doklady 9:806-807, 1968.

[21] Jě̌́́BEK, E., Canonical rules, The Journal of Symbolic Logic 74(4):1171-1205, 2009.

[22] Maksimova, L. L., On maximal intermediate logics with the disjunction property, Studia Logica 45(1):69-75, 1986.

[23] Priestley, H. A., Representation of distributive lattices by means of ordered Stone spaces, Bulletin of the London Mathematical Society 2:186-190, 1970.

[24] Priestley, H. A., Ordered topological spaces and the representation of distributive lattices, Proceedings of the London Mathematical Society 24:507-530, 1972.

[25] Priestley, H. A., The construction of spaces dual to pseudocomplemented distributive lattices, Quarterly Journal of Mathematics, Oxford Series 26(102):215-228, 1975.

[26] Zakharyaschev, M., Syntax and semantics of superintuitionistic logics, Algebra and Logic 28(4):262-282, 1989.

[27] Zakharyaschev, M., Canonical formulas for K4. Basic results, The Journal of Symbolic Logic 57(4):1377-1402, 1992.

G. Bezhanishvili

Department of Mathematical Sciences

New Mexico State University

Las Cruces NM 88003, USA

guram@math.nmsu.edu

N. Bezhanishvili, J. Ilin

Institute for Logic, Language and Computation

University of Amsterdam

P.O. Box 94242

1090 GE Amsterdam, The Netherlands

N.Bezhanishvili@uva.nl

J. Ilin

ilin.juli@gmail.com 